\title{
HISTORY OF GALAXY INTERACTIONS AND THEIR IMPACT ON STAR FORMATION OVER THE LAST 7 Gyr
} FROM GEMS

\author{
Shardha Joget ${ }^{1}$, Sarah H. Miller ${ }^{1,2}$, Kyle Penner $^{1,3}$, Rosalind E. Skelton $^{4}$, Christopher J. Conselice $^{5}$, \\ Rachel S. Somerville ${ }^{4}$, Eric F. Bell ${ }^{4}$, Xian Zhong Zheng ${ }^{6}$, Hans-Walter Rix ${ }^{4}$, Aday R. Robaina ${ }^{4}$, Fabio D. Barazza ${ }^{7}$, \\ Marco Barden $^{8}$, Andrea Borch ${ }^{4}$, Steven V. W. Beckwith ${ }^{9}$, John A. R. Caldwell ${ }^{10}$, Chien Y. Peng ${ }^{11}$, \\ Catherine Heymans ${ }^{12}$, Daniel H. McIntosh ${ }^{13}$, Boris HäußLer ${ }^{4}$, Knud Jahnke ${ }^{4}$, Klaus Meisenheimer ${ }^{4}$, \\ Sebastian F. Sanchez ${ }^{14}$, Lutz Wisotzki ${ }^{15}$, Christian Wolf $^{2}$, and Casey Papovich ${ }^{16}$ \\ ${ }^{1}$ Department of Astronomy, University of Texas at Austin, 1 University Station C1400, Austin, TX 78712-0259, USA; sj@astro.as.utexas.edu \\ ${ }^{2}$ Astrophysics, University of Oxford, Keble Road, Oxford OX1 3RH, UK \\ ${ }^{3}$ Department of Astronomy, University of Arizona, Steward Observatory, 933 N. Cherry Avenue, Tucson, AZ 85721, USA \\ ${ }^{4}$ Max-Planck-Institut für Astronomie, Königstuhl 17, D-69117, Heidelberg, Germany \\ ${ }^{5}$ School of Physics and Astronomy, The University of Nottingham, University Park, Nottingham NG7 2RD, UK \\ ${ }^{6}$ Purple Mountain Observatory, West Beijing Road 2, Nanjing, 210008, China \\ ${ }^{7}$ Laboratoire d'Astrophysique, École Polytechnique Fédérale de Lausanne (EPFL), Observatoire, 1290 Sauverny, Switzerland \\ ${ }^{8}$ Institute for Astro- and Particle Physics, University of Innsbruck, Technikerstr. 25/8, A-6020 Innsbruck, Austria \\ ${ }^{9}$ Department of Physics and Astronomy, Johns Hopkins University, Charles and 4th Street, Baltimore, MD 21218, USA \\ ${ }^{10}$ University of Texas, McDonald Observatory, Fort Davis, TX 79734, USA \\ ${ }^{11}$ NRC Herzberg Institute of Astrophysics, Victoria, Canada \\ ${ }^{12}$ SUPA, Institute for Astronomy, University of Edinburgh, Royal Observatory, Edinburgh EH9 3HJ, UK \\ 13 Department of Astronomy, University of Massachusetts, 710 North Pleasant Street, Amherst, MA 01003, USA \\ ${ }^{14}$ Centro Astronómico Hispano Alemán, Calar Alto, CSIC-MPG, C/Jesús Durbán Remón 2-2, E-04004 Almeria, Spain \\ 15 Astrophysikalisches Institut Potsdam, An der Sternwarte 16, D-14482 Potsdam, Germany \\ ${ }^{16}$ George P. and Cynthia W. Mitchell Institute for Fundamental Physics and Astronomy, and Department of Physics, Texas A\&M University, College Station, TX \\ 77843-4242, USA \\ Received 2008 June 30; accepted 2009 March 20; published 2009 May 18
}

\begin{abstract}
We perform a comprehensive estimate of the frequency of galaxy mergers and their impact on star formation over $z \sim 0.24-0.80$ (lookback time $\left.T_{\mathrm{b}} \sim 3-7 \mathrm{Gyr}\right)$ using $\sim 3600\left(M \geqslant 1 \times 10^{9} M_{\odot}\right)$ galaxies with GEMS Hubble Space Telescope, COMBO-17, and Spitzer data. Our results are as follows. (1) Among $\sim 790$ high-mass $(M \geqslant 2.5 \times$ $10^{10} M_{\odot}$ ) galaxies, the visually based merger fraction over $z \sim 0.24-0.80$, ranges from $9 \% \pm 5 \%$ to $8 \% \pm 2 \%$. Lower limits on the major merger and minor merger fraction over this interval range from $1.1 \%$ to $3.5 \%$, and $3.6 \%$ to $7.5 \%$, respectively. This is the first, albeit approximate, empirical estimate of the frequency of minor mergers over the last $7 \mathrm{Gyr}$. Assuming a visibility timescale of $\sim 0.5 \mathrm{Gyr}$, it follows that over $T_{\mathrm{b}} \sim 3-7 \mathrm{Gyr}, \sim 68 \%$ of high-mass systems have undergone a merger of mass ratio $>1 / 10$, with $\sim 16 \%, 45 \%$, and $7 \%$ of these corresponding respectively to major, minor, and ambiguous "major or minor" mergers. The average merger rate is $\sim$ a few $\times 10^{-4}$ galaxies Gyr $^{-1}$ $\mathrm{Mpc}^{-3}$. Among $\sim 2840$ blue-cloud galaxies of mass $M \geqslant 1.0 \times 10^{9} M_{\odot}$, similar results hold. (2) We compare the empirical merger fraction and merger rate for high-mass galaxies to three $\Lambda$ cold dark matter-based models: halo occupation distribution models, semi-analytic models, and hydrodynamic SPH simulations. We find qualitative agreement between observations and models such that the (major+minor) merger fraction or rate from different models bracket the observations, and show a factor of 5 dispersion. Near-future improvements can now start to rule out certain merger scenarios. (3) Among $3698 M \geqslant 1.0 \times 10^{9} M_{\odot}$ galaxies, we find that the mean star formation rate (SFR) of visibly merging systems is only modestly enhanced compared to non-interacting galaxies over $z \sim$ $0.24-0.80$. Visibly merging systems only account for a small fraction $(<30 \%)$ of the cosmic SFR density over $T_{\mathrm{b}} \sim$ 3-7 Gyr. This complements the results of Wolf et al. over a shorter time interval of $T_{\mathrm{b}} \sim 6.2-6.8 \mathrm{Gyr}$, and suggests that the behavior of the cosmic SFR density over the last $7 \mathrm{Gyr}$ is predominantly shaped by non-interacting galaxies.
\end{abstract}

Key words: galaxies: evolution - galaxies: fundamental parameters - galaxies: kinematics and dynamics galaxies: structure

Online-only material: color figures

\section{INTRODUCTION}

Hierarchical $\Lambda$ cold dark matter $(\Lambda \mathrm{CDM})$ models provide a successful paradigm for the growth of dark matter on large scales. The evolution of galaxies within $\Lambda$ CDM cosmogonies depends on the baryonic merger history, the star formation (SF) history, the nature and level of feedback from supernovae and active galactic nuclei (AGNs), the redistribution of angular momentum via bars or mergers, and other aspects of the baryonic physics. Empirical constraints on the fate of the baryonic component, in particular their merger and SF history, are key for developing a coherent picture of galaxy evolution and testing galaxy evolution models (e.g., Kauffmann et al. 1993; Somerville \& Primack 1999; Navarro \& Steinmetz 2000; Murali et al. 2002; Governato et al. 2004; Springel et al. 2005a, 2005b; Maller et al. 2006). Such constraints can help to resolve several major areas of discord between observations and $\Lambda \mathrm{CDM}$ models of galaxy evolution, such as the angular momentum crisis, the problem of bulgeless and low bulge-to-total $(B / T)$ ratio galaxies (Navarro \& Benz 1991; D’Onghia \& Burkert 2004; Kautsch 
et al. 2006; Barazza et al. 2008; Weinzirl et al. 2009), and the substructure problem.

The merger history of galaxies impacts the mass assembly, SF history, AGN activity, and structural evolution of galaxies. Yet, the merger rate has proved hard to robustly measure for a variety of reasons. Initially, small samples hindered the first efforts to measure merger rates (Patton et al. 2000; Le Fèvre et al. 2000; Conselice et al. 2003). Later studies drew from larger samples, and have used a variety of methods to characterize the interaction history of galaxies at $z<1$. Studies based on close pairs report a major merger fraction of $\sim 2 \%-10 \%$ over $z \sim 0.2-1.2$ (Lin et al. 2004; Kartaltepe et al. 2007; de Ravel et al. 2008) and $5 \%$ for massive galaxies at $z \sim 0.4-0.8$ (Bell et al. 2006).

Among these studies, the estimated fraction of galaxies, which have experienced a major merger since $z \sim 1.2$ differ. Lin et al. (2004) find that $\sim 9 \%$ of the massive early type galaxies experienced a major merger since $z \sim 1.2$, while Bell et al. (2006) report that $\sim 50 \%$ of all galaxies with present-day stellar masses above $5 \times 10^{10} M_{\odot}$ have undergone a major merger since $z \sim 0.8$. This difference of a factor of 5 between the two studies is addressed by Bell et al. (2006; see their footnote 9) and is traced primarily to differences in the following factors: the data set used, the redshift integration method, the way in which the authors handle the relative number density of the parent population from which pairs are drawn and that of the remnant population, the assumed visibility timescale, and the fraction of pairs estimated to be true gravitationally bound pairs.

Studies based on Gini-M20 coefficients report a fairly constant fraction $(\sim 7 \pm 0.2 \%)$ of disturbed galaxies over $z \sim 0.2-$ 1.2 among bright galaxies (Lotz et al. 2008) in the AEGIS survey. Similar trends are found from early results based on visual classification and asymmetry parameters of high-mass galaxies (e.g., Jogee et al. 2008). The study by Cassata et al. (2005) based on both pairs and asymmetries report a mild increase in the merger rate with redshift up to $z \sim 1$, with the caveat of a small sample size. The merger rate/fraction at $z>1$ remains highly uncertain, owing to relatively modest volumes and bandpass shifting effects, but there is general trend toward higher merger fractions at higher redshifts (e.g., Conselice et al. 2003; Cassata et al. 2005).

Studies to date have brought important insights but face several limitations. In the case of studies based on close (separation $\sim 5-30 \mathrm{kpc}$ ) pairs, the translation of the pair frequency into a merger rate is somewhat uncertain due to several factors. The uncertainties in the spectrophotometric redshifts for some of the galaxies in pairs can cause us to overestimate or underestimate the true close pair fraction, with the latter effect being more likely. Corrections for this effect are uncertain and depend on the shape of the spectrophotometric redshift errors (e.g., see Bell et al. 2006 for discussion). Secondly, even pairs with members at the same redshift may not be gravitationally bound, and may therefore not evolve into a merger in the future: this effect causes the close pair fraction to be upper limits for the merger fraction. Thirdly, gravitationally bound pairs captured by this method sample different phases of an interaction depending on the separation, and any merger rate inferred depends on the separation, orbital eccentricity, and orbital geometry.

In the case of studies, which use automated parameters, such as CAS asymmetry $A$ and clumpiness parameters (Conselice et al. 2000; Conselice 2003) or Gini-M20 coefficients (e.g., Lotz et al. 2004) to identify merging galaxies can fail to pick stages of both major and minor mergers where distortions do not dominate the total light contribution (Section 3.4). Comparison with simulations suggest that the CAS criterion $(A>0.35$ and $A>S$; Conselice 2003) capture major mergers about $1 / 3$ of the time, while the eye is sensitive to major merger features over twice as long (e.g., Conselice 2006; Section 3.4). To complicate matters, automated asymmetry parameters can also capture noninteracting galaxies hosting small-scale asymmetries that are produced by stochastic SF (Section 4.2). Thus, it is important to use several methods to assess the robustness of results and identify the systematics.

In this paper, we present a complementary study of the frequency of mergers and their impact on the SF activity of galaxies over $z \sim 0.24-0.80$ (lookback times $T_{\mathrm{b}}$ of 3-7 $\mathrm{Gyr}^{17}$ ) using Hubble Space Telescope Advanced Camera for Surveys (HST ACS), COMBO-17, and Spitzer $24 \mu \mathrm{m}$ data of $\sim 3600$ galaxies in the Galaxy Evolution from Morphology and SEDS (GEMS) survey. The outline of the paper is given below and describes how this study complements existing work:

1. We use a large sample of $\sim 3600\left(M \geqslant 1 \times 10^{9} M_{\odot}\right)$ galaxies to get robust number statistics for the merger fraction among $\sim 790$ high-mass $\left(M \geqslant 2.5 \times 10^{10} M_{\odot}\right)$ galaxies and $\sim 2840 M \geqslant 1 \times 10^{9} M_{\odot}$ blue-cloud galaxies (Section 2, Table 1, Table 2).

2. Two independent methods are used to identify merging galaxies: a physically driven visual classification system complemented with spectrophotometric redshifts and stellar masses (Sections 3.2 to 3.3), as well as automated CAS asymmetry and clumpiness parameters (Section 3.4). This allows one of the most extensive comparisons to date between CAS-based and visual classification results (Section 4.2).

3. We design the visual classification system in a way that allows merger fractions and rates from observations and theoretical models to be readily compared. We classify as mergers those systems that show evidence of having experienced a merger of mass ratio $>1 / 10$ within the last visibility timescale. Throughout this paper, we use the standard definition whereby major mergers are defined to have stellar mass ratio $(1 / 4<M 1 / M 2 \leqslant 1 / 1)$, while minor mergers have $(1 / 10<M 1 / M 2 \leqslant 1 / 4)$. We set lower limits on the major and minor merger fraction (Section 4.1). To our knowledge, this is the first, albeit approximate, empirical estimate of the frequency of minor mergers over the last 7 Gyr. While many earlier studies focused on major mergers, it is important to constrain minor mergers as well, since they dominate the merger rates in $\Lambda$ CDM models, and play an important role in building the bulges of massive galaxies (e.g., Weinzirl et al. 2009).

4. We compare the empirical merger fraction and rate to a suite of $\Lambda \mathrm{CDM}$-based simulations of galaxy evolution, including halo occupation distribution models, semi-analytic models (SAMs), and hydrodynamic SPH simulations (Section 4.5). To our knowledge, these extensive comparisons have not been attempted to date, and are long overdue.

5. The idea that galaxy interactions generally enhance the star formation rate (SFR) of galaxies is well established from observations (e.g., Larson \& Tinsley 1978; Joseph \& Wright 1985; Kennicutt et al. 1987; Barton Gillespie et al. 2003) and simulations (e.g., Negroponte \& White 1983; Hernquist 1989; Barnes \& Hernquist 1991, 1996; Mihos \& Hernquist 1994, 1996; Springel et al. 2005b). However, simulations

\footnotetext{
17 We assume a flat cosmology with $\Omega_{\mathrm{m}, 0}=1-\Omega_{\lambda}=0.3$ and $H_{0}=70 \mathrm{~km}$ $\mathrm{s}^{-1} \mathrm{Mpc}^{-1}$ throughout.
} 
cannot uniquely predict the factor by which galaxy mergers enhance the SF activity of galaxies over the last $7 \mathrm{Gyr}$, since both the SFR and properties of the remnants in simulations are highly sensitive to the stellar feedback model, the bulgeto-disk $(B / D)$ ratio, the gas mass fractions, and orbital geometry (e.g., Cox et al. 2006; di Matteo et al. 2007). This motivates us in Section 4.6 to empirically investigate the impact of interactions on the average UV-based and UV+IR-based SFR of high-mass $\left(M \geqslant 2.5 \times 10^{10} M_{\odot}\right)$ and intermediate-to-high-mass $\left(M \geqslant 1 \times 10^{9} M_{\odot}\right)$ galaxies over $z \sim 0.24-0.80$.

6 . The SF properties of merging and non-interacting galaxies since $z<1$ is of great astrophysical interest, given that the cosmic SFR density is claimed to decline by a factor of 4-10 since $z \sim 1$ (e.g., Lilly et al. 1996; Ellis et al. 1996; Flores et al. 1999; Haarsma et al. 2000; Hopkins 2004; Pérez-González et al. 2005; Le Floc'h et al. 2005). In Section 4.7, we set quantitative limits on the contribution of visibly merging systems to the UV-based and UV+IR-based SFR density over $z \sim 0.24-0.80$. Our study covers a $4 \mathrm{Gyr}$ interval $\left(T_{\mathrm{b}} \sim 3-7\right.$ Gyr or $\left.z \sim 0.24-0.80\right)$ and extends the earlier studies carried out over a smaller $0.6 \mathrm{Gyr}$ interval $\left(T_{\mathrm{b}} \sim 6.2-6.8\right.$ Gyr or $\left.z \sim 0.65-0.75\right)$ by Wolf et al. (2005) and Bell et al. (2005) on the UV and IR luminosity density, respectively. Our study also complements IR-based studies by Lotz et al. (2008), Hammer et al. (2005; 195 galaxies at $z>0.4)$ and Melbourne et al. (2005; $\sim 800$ galaxies) in terms of better number statistics and the use of both UV-based and IR-based SFR indicators.

\section{DATA SET AND SAMPLE SELECTION}

This study uses data from the GEMS (Rix et al. 2004) survey, which provides high-resolution HST ACS images in the F606W and F850LP filters over an $800 \operatorname{arcmin}^{2}\left(\sim 28^{\prime} \times 28^{\prime}\right)$ field centered on the Chandra Deep Field-South (CDF-S). Accurate spectrophotometric redshifts $\left[\delta_{z} /(1+z) \sim 0.02\right.$ down to $\left.R_{\text {Vega }}=24\right]$ and spectral energy distributions (SEDs), based on 5 broad bands (UBVRI) and 12 medium band filters, are available from the COMBO-17 project (Wolf et al. 2004). The ACS data reach a limiting $5 \sigma$ depth for point sources of 28.3 and 27.1 $\mathrm{AB}$ mag in F606W and F850LP, respectively (Rix et al. 2004; Caldwell et al. 2008). The effective point spread function (PSF) in a single F606W image is $\sim 0.07$, corresponding to $260 \mathrm{pc}$ at $z \sim 0.24$ and $520 \mathrm{pc}$ at $z \sim 0.80$. The PSF of combined drizzle images is $\sim 0^{\prime \prime} 1$. In addition to HST ACS imaging, the GEMS field has panchromatic coverage which includes Spitzer (Rieke et al. 2004; Papovich et al. 2004) and Chandra data (Alexander et al. 2003; Lehmer et al. 2005).

We use stellar masses from Borch et al. (2006). We refer the reader to the latter publication for a detailed description and provide a summary here. Using the 17-passband photometry from COMBO-17, objects were classified as main sequence, stars, white dwarfs, galaxies, and quasars using color indices and their photometric redshifts were estimated using simple dust-reddened single-burst SED templates (Wolf et al. 2004). For galaxies and quasars, the joint probability of a given redshift and a given rest-frame SED is derived and this procedure provides a minimum error variance estimation of both the redshift and the SED template. Once the redshfit has been estimated, the SEDs in 17 bands were fitted with a new set of template SEDs with more plausible SF histories in order to derive a stellar $M / L$ (Borch et al. 2006). The library of SEDs is built using the PEGASE stellar population synthesis model (see Fioc \& Rocca-Volmerange 1997 for an earlier version of the model) and the underlying SF histories are parameterized by the three-component model, with a Kroupa (Kroupa et al. 1993) initial mass function (IMF) adopted in the mass regime 0.1-120 $M_{\odot}$. We note that the stellar masses are consistent within $10 \%$ with the masses that would be estimated using a different Kroupa (2001) or Chabrier (2003) IMF. ${ }^{18}$ The reddest templates have smoothly varying exponentially declining SF episodes, intermediate templates have a contribution from a low-level constant level of SF, while the bluer templates have a recent burst of SF superimposed. Random stellar mass errors are $<0.3$ dex on a galaxy-by-galaxy basis, and systematic errors in the stellar masses were argued to be at the 0.1 dex level (Borch et al. 2006). Bell \& de Jong (2001) argued that galaxies with large bursts of recent SF could drive down stellar M/L values by up to $0.5 \mathrm{dex}$ at a given color, but we note that the Borch et al. (2006) templates do include bursts explicitly, thus compensating for the worst of the uncertainties introduced by bursting SF histories.

We present the results based on the visual classification and CAS (Conselice 2003) parameters (Section 3) of GEMS F606W, rather than F850LP images, for the following reasons. The F606W images are $\sim 1.2 \mathrm{AB}$ magnitude deeper than the GEMS F850LP images and allow more reliable characterization of morphological features in the presence of cosmological surface brightness dimming at the rate of $(1+z)^{-4}$ (e.g., Barden et al. 2008). Furthermore, the low signal to noise in the F850LP images leads to large error bars on the asymmetry $A$ and clumpiness $S$ parameters generated by the CAS code, effectively making it impractical to use these values in CAS merger diagnostics (Section 3.4). When using the F606W images, we only include results over the redshift range $z \sim 0.24-0.80$ in order to ensure that the rest-frame wavelength $\lambda_{\text {rest }}$ stays primarily in the optical band and does not shift well below the $4000 \AA$ break. In the fourth redshift bin $(z \sim 0.6-0.8) \lambda_{\text {rest }}$ shifts to the violet/near-UV (3700 $-3290 \AA$ ), but as we show in Section 4.1, this does not significantly impact the results. We discard the last redshift bin at $z>0.8$ where $\lambda_{\text {rest }}$ shifts into the far-UV. These steps lead to a sample of 4766 galaxies selected down to $R_{\text {Vega }} \leqslant 24$, over $z \sim 0.24-0.80$ ( $\left.T_{\text {back }} \sim 3-7 \mathrm{Gyr}\right)$.

In this paper, we present results for two samples of astrophysical interest, which are derived by applying stellar mass cuts to the above sample of $\sim 4766$ galaxies The first sample (henceforth sample S1) focuses on galaxies with high stellar mass $\left(M \geqslant 2.5 \times 10^{10} M_{\odot}\right.$; Table 1$)$. The sample size is originally 804 galaxies, out of which 798 (99.2\%) could be visually classified. For this stellar mass range, the red-sequence and bluecloud galaxies are both complete out to the highest redshift bin $z \sim 0.62-0.80$ for our sample, and we have theoretical predictions for comparison (see Section 4.5) from semianalytical models (e.g, Somerville et al. 2008; Hopkins et al. 2008; Khochfar \& Burkert 2005; Bower et al. 2006), $N$-body (D'Onghia et al. 2008), and hydrodynamical SPH simulations (e.g., Maller et al. 2006). Note that the survey has few galaxies above $10^{11} M_{\odot}$ (Figure 1), and hence the high-mass $\left(M \geqslant 2.5 \times 10^{10} M_{\odot}\right)$ sample primarily involves galaxies in the range $2.5 \times 10^{10}$ to $10^{11} M_{\odot}$.

We also present selected results for the sample S2 of 3698 galaxies with $M \geqslant 1 \times 10^{9} M_{\odot}$ and visual classes. Although this sample includes the $\sim 790$ high-mass galaxies in sample

\footnotetext{
18 We adopt a Chabrier (2003) IMF when exploring the contribution of mergers to the cosmic SFR (Sections 4.6 and 4.7).
} 
Table 1

Visual Merger Fraction for High-Mass $\left(M \geqslant 2.5 \times 10^{10} M_{\odot}\right)$ Sample $\mathrm{S} 1[N=789]$

\begin{tabular}{|c|c|c|c|c|c|}
\hline (1) & Redshift bin & 1 & 2 & 3 & 4 \\
\hline (2) & Redshift range & $0.24-0.34$ & $0.34-0.47$ & $0.47-0.62$ & $0.62-0.80$ \\
\hline (3) & Lookback time [Gyr] & $3.0-4.0$ & $4.0-5.0$ & $5.0-6.0$ & $6.0-7.0$ \\
\hline (4) & $\lambda_{\text {rest }}$ in F606W [ $\left.\mathrm{A}\right]$ & $4470-4414$ & $4414-4023$ & $4023-3651$ & $3651-3286$ \\
\hline (5) & Total no of galaxies & 46 & 84 & 213 & 446 \\
\hline (6) & Fraction of mergers & $0.087 \pm 0.047$ & $0.083 \pm 0.037$ & $0.089 \pm 0.030$ & $0.0807 \pm 0.025$ \\
\hline (6a) & Lower limit on major merger fraction & $0.022 \pm 0.021$ & $0.035 \pm 0.022$ & $0.014 \pm 0.009$ & $0.011 \pm 0.006$ \\
\hline (6b) & Lower limit on minor merger fraction & $0.065 \pm 0.040$ & $0.036 \pm 0.022$ & $0.075 \pm 0.027$ & $0.049 \pm 0.016$ \\
\hline$(6 c)$ & Fraction of ambiguous minor/major mergers & 0.00 & $0.012 \pm 0.012$ & 0.00 & $0.020 \pm 0.008$ \\
\hline (7) & Fraction of non-interacting E-Sd & $0.913 \pm 0.241$ & $0.869 \pm 0.229$ & $0.878 \pm 0.229$ & $0.785 \pm 0.205$ \\
\hline (8) & Fraction of non-interacting Irr1 & 0.000 & $0.024 \pm 0.018$ & $0.009 \pm 0.007$ & $0.025 \pm 0.010$ \\
\hline (9) & Fraction of compact & 0.000 & $0.024 \pm 0.018$ & $0.023 \pm 0.012$ & $0.11 \pm 0.032$ \\
\hline
\end{tabular}

Notes. Rows are: (1) redshift bin; (2) range in redshift covered by the bin; (3) range in lookback time covered by the bin; (4) range in rest-frame wavelength traced by the F606W filter over the bin, assuming a pivot wavelength of $5915 \AA$; $(5)$ total number of high-mass galaxies per bin; (6) fraction of systems with evidence of a recent merger of mass ratio $>1 / 10$. These include both major $(M 1 / M 2 \geqslant 1 / 4)$ and minor $(1 / 10<M 1 / M 2 \leqslant 1 / 4)$ mergers; $(6 a)$ lower limit on the fraction of galaxies undergoing major mergers; (6b) lower limit on the fraction of galaxies undergoing minor mergers; (6c) remaining fraction of galaxies that could be either major or minor mergers; (7-9) fraction of non-interacting E-Sd, non-interacting Irr1, and compact systems.

Table 2

Visual Merger Fraction for $M \geqslant 1.0 \times 10^{9} M_{\odot}$ Sample S2 $[N=3698]$

\begin{tabular}{|c|c|c|c|c|c|}
\hline (1) & Redshift bin & 1 & 2 & 3 & 4 \\
\hline (2) & Redshift range & $0.24-0.34$ & $0.34-0.47$ & $0.47-0.62$ & $0.62-0.80$ \\
\hline (3) & Lookback time [Gyr] & $3.0-4.0$ & $4.0-5.0$ & $5.0-.6-0$ & $6.0-7.0$ \\
\hline (4) & $\lambda_{\text {rest }}$ in F606W [ $\left.\mathrm{\AA}\right]$ & $4470-4414$ & $4414-4023$ & $4023-3651$ & $3651-3286$ \\
\hline \multicolumn{6}{|c|}{ All $[N=3698]$} \\
\hline (5) & Total no of galaxies & 235 & 480 & 1117 & 1866 \\
\hline (6) & Fraction of mergers & $0.111 \pm 0.035$ & $0.090 \pm 0.027$ & $0.074 \pm 0.021$ & $0.062 \pm 0.017$ \\
\hline (6a) & Lower limit on major merger fraction & $0.013 \pm 0.008$ & $0.006 \pm 0.004$ & $0.004 \pm 0.002$ & $0.009 \pm 0.003$ \\
\hline$(6 b)$ & Lower limit on minor merger fraction & $0.038 \pm 0.016$ & $0.021 \pm 0.008$ & $0.021 \pm 0.007$ & $0.017 \pm 0.005$ \\
\hline$(6 c)$ & Fraction of ambiguous minor/major mergers & $0.060 \pm 0.022$ & $0.062 \pm 0.020$ & $0.048 \pm 0.014$ & $0.036 \pm 0.010$ \\
\hline (7) & Fraction of non-interacting E-Sd & $0.850 \pm 0.220$ & $0.846 \pm 0.220$ & $0.796 \pm 0.207$ & $0.793 \pm 0.206$ \\
\hline (8) & Fraction of non-interacting Irr1 & $0.064 \pm 0.023$ & $0.052 \pm 0.017$ & $0.108 \pm 0.030$ & $0.064 \pm 0.018$ \\
\hline (9) & Fraction of compact & 0.000 & $0.012 \pm 0.006$ & $0.021 \pm 0.007$ & $0.080 \pm 0.022$ \\
\hline \multicolumn{6}{|c|}{ Blue Cloud $[N=2844]$} \\
\hline (10) & Total no of galaxies & 154 & 332 & 876 & 1482 \\
\hline (11) & Fraction of mergers & $0.149 \pm 0.048$ & $0.114 \pm 0.034$ & $0.088 \pm 0.025$ & $0.069 \pm 0.019$ \\
\hline (11a) & Lower limit on major merger fraction & $0.013 \pm 0.009$ & 0.00 & $0.005 \pm 0.003$ & $0.008 \pm 0.003$ \\
\hline (11b) & Lower limit on minor merger fraction & $0.046 \pm 0.020$ & $0.024 \pm 0.010$ & $0.023 \pm 0.008$ & $0.016 \pm 0.005$ \\
\hline (11c) & Fraction of ambiguous minor/major mergers & $0.091 \pm 0.033$ & $0.090 \pm 0.028$ & $0.060 \pm 0.018$ & $0.046 \pm 0.013$ \\
\hline$(12)$ & Fraction of non-interacting E-Sd & $0.753 \pm 0.199$ & $0.801 \pm 0.209$ & $0.753 \pm 0.196$ & $0.784 \pm 0.204$ \\
\hline (13) & Fraction of non-interacting Irr1 & $0.097 \pm 0.035$ & $0.075 \pm 0.024$ & $0.136 \pm 0.037$ & $0.080 \pm 0.022$ \\
\hline (14) & Fraction of compact & 0.000 & $0.009 \pm 0.006$ & $0.023 \pm 0.008$ & $0.067 \pm 0.018$ \\
\hline
\end{tabular}

Notes. Rows are: (1)-(9) As in Table 1, but for intermediate-mass $\left(M \geqslant 1.0 \times 10^{9} M_{\odot}\right)$ galaxies. However, note that the intermediate-mass sample is incomplete for the red sequence. (10)-(14) Ditto, but for the blue cloud, where the sample is complete.

$\mathrm{S} 1$, it is dominated by systems of intermediate mass $\left(10^{9} \leqslant\right.$ $\left.M / M_{\odot}<2.5 \times 10^{10}\right)$. For the mass range $M \geqslant 1 \times 10^{9} M_{\odot}$, the blue cloud is complete in our sample out to $z \sim 0.80$, while the red sequence is incomplete in the higher redshift bins. Where appropriate, we will therefore present results for the bluecloud sample only (e.g., lower part of table 2). The rest-frame $U-V$ color is plotted versus the stellar mass for the sample S2 in Figure 1. The redshift interval is divided into four $1 \mathrm{Gyr}$ bins. The diagonal line marks the separation of the red-sequence and the blue-cloud galaxies (BCG) at the average redshift $z_{\text {ave }}$ of the bin. We use the definition in Borch et al. (2006) and Bell et al. (2004) for CDF-S:

$$
(U-V)_{\text {rest }}>0.227 \log \left(M / M_{\odot}\right)-1.26-0.352 z .
$$

The vertical lines in Figure 1 marks the mass completeness limit (Borch et al. 2006) for the red-sequence galaxies. The blue-cloud galaxies are complete well below this mass (Borch et al. 2006).

\section{METHODOLOGY: IDENTIFYING MERGERS AND NON-INTERACTING GALAXIES}

\subsection{Overview of the Methodology}

Galaxy mergers with a mass ratio $M 1 / M 2>1 / 10$ can have a significant impact on galaxy evolution. According to simulations, major mergers (defined as those with mass ratio $1 / 4$ $<M 1 / M 2 \leqslant 1 / 1)$ typically destroy stellar disks, transforming them via violent relaxation, into systems with a steep or $r^{1 / 4}$ 


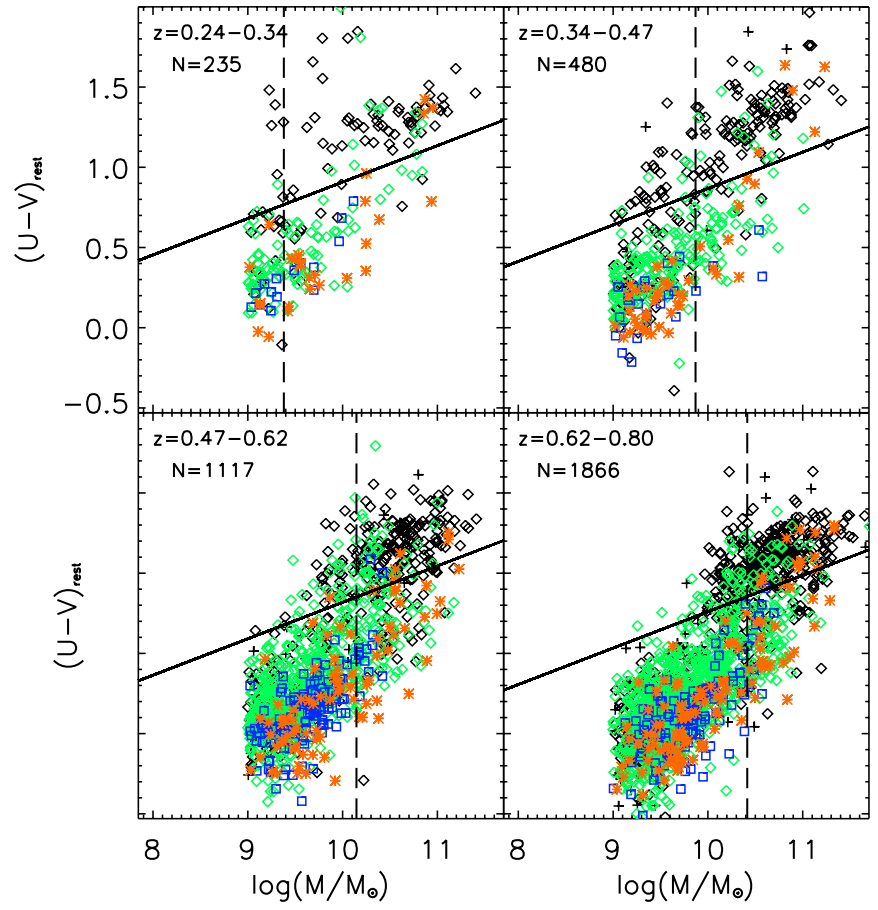

Figure 1. Rest-frame $U-V$ color is plotted vs. the stellar mass over $z \sim 0.24$ 0.80 for the sample S2 of $\sim 3698$ galaxies with $M \geqslant 1 \times 10^{9} M_{\odot}$. The four panels denote the four redshift bins, which span $1 \mathrm{Gyr}$ each, and cumulatively cover the interval $z \sim 0.24-0.80$ ( $\left.T_{\text {back }} \sim 3-7 \mathrm{Gyr}\right) . N$ denotes the number of galaxies in each bin. The diagonal line marks the separation of the red-sequence galaxies and the blue-cloud galaxies at the average redshift $z_{\text {ave }}$ of the bin. The vertical lines marks the mass completeness limit (Borch et al. 2006) for the red-sequence galaxies, while the blue-cloud galaxies are complete well below this mass. For the mass range $M \geqslant 1 \times 10^{9} M_{\odot}$, the blue cloud is complete in our sample out to $z \sim 0.80$, while the red sequence is incomplete in the higher redshift bins. Galaxies are coded according to their visual type (VT) in the F606W band: "Mergers" (orange stars), "Non-Interacting E+S0+Sa" (black diamonds), "Non-Interacting Sb-Sc + Sd" (green diamonds), and "Non-Interacting Irr1" (blue squares).

(A color version of this figure is available in the online journal.)

de Vaucouleurs-type stellar profile, such as ellipticals (e.g., Negroponte \& White 1983; Barnes \& Hernquist 1991; Mihos \& Hernquist 1996; Struck 1997; Naab \& Burkert 2001; but see Robertson et al. 2004). Simulations further suggest that recent major mergers at $z<1$ are typically associated with arcs, shells, ripples, tidal tails, large tidal debris, highly asymmetric light distributions, double nuclei inside a common body, galaxies linked via tidal bridges of light, and galaxies enclosed within the same distorted envelope of light.

Minor mergers (defined as those with $1 / 10<M 1 / M 2 \leqslant$ 1/4) of two spirals will not destroy the disk of the larger spiral (e.g., Hernquist \& Mihos 1995; Smith 1997; Jogee et al. 1999). Typically, the smaller companion sinks via dynamical friction, may excite warps, bars, spirals, and other non-axisymmetric perturbations, and leads to vertical heating, arcs, shells, ripples, tidal tails, tidal debris, warps, offset rings, highly asymmetric light distributions, etc (e.g., Quinn et al. 1993; Hernquist \& Mihos 1995; Mihos et al. 1995; Quinn et al. 1993; Smith 1994; Jogee 1999; Jogee et al. 1998, 1999; review by Jogee 2006 and references therein).

One goal of this paper is to identify systems whose morphology and other properties suggest they have recently experienced a merger of mass ratio $M 1 / M 2>1 / 10$. We employ two methods: a physically driven visual classification system (Sections 3.2-3.3) complemented with spectrophotometric redshifts and stellar masses, and a method based on quantitative asymmetry $(A)$, and clumpiness $(S)$ parameters (Section 3.4) derived using the CAS code (Conselice 2003). While many studies use only automated methods or visual classification, we choose to use both methods in order to better assess the systematics, and to test the robustness of our results.

\subsection{Visual Classification of Mergers}

The visual classification system we adopted for identifying mergers was aimed at setting a procedural framework that allows merger fractions and rates from observations and the theoretical models (outlined in Section 4.5) to be defined in similar ways and to be readily compared.

Specifically the theoretical models described in Section 4.5 track systems which have a stellar mass $M_{*} \geqslant M_{\text {cut }}$ and have experienced a merger of a certain mass ratio $M 1 / M 2$ within the last visibility timescale $t_{\mathrm{vis}}$, between times $\left(t_{\mathrm{obs}}-t_{\mathrm{vis}}\right)$ and $t_{\mathrm{obs}}$. Here $t_{\mathrm{obs}}$ is the time corresponding to the observed redshift $z$; $t_{\text {vis }}$ is the timescale over which morphological distortions remain visible after a merger and we adopt a nominal value of $0.5 \mathrm{Gyr}$ (see Section 4.3); $M_{\text {cut }}$ is the cutoff mass for the merger, which is $2.5 \times 10^{10} M_{\odot}$ for the high-mass sample S1 and $1 \times 10^{9}$ $M_{\odot}$ for the intermediate-mass sample S2 (Section 2). In the models, major mergers are associated with a mass ratio of $1 / 4$ $<M 1 / M 2 \leqslant 1 / 1$, minor mergers with $1 / 10<M 1 / M 2 \leqslant 1 / 4$, and major+minor mergers with $M 1 / M 2>1 / 10$.

Analogous to the theoretical models, the goal of the visual classification system is to identify systems with $M_{*} \geqslant M_{\text {cut }}$, which show evidence of having experienced a merger of mass ratio $>1 / 10$ within the last visibility timescale $t_{\text {vis }}$. In this paper, we refer to such systems either as interacting galaxies or as mergers. These mergers are denoted as having visual type "Int" in the figures of this paper and examples are shown in Figure 2. In practice, they consist of three types of mergers, which we denote as Int-1, Int-2a, and Int-2b, and define in Sections 3.2.1 and 3.2.2. These three types of systems encompass young to advanced mergers, and are identified/handled in different ways, as described below.

\subsubsection{Mergers of type Int-1}

Mergers of type Int-1 primarily represent advanced mergers of mass $M_{*} \geqslant M_{\text {cut }}$, which appear as a single system in ACS images, and are likely associated with a merger of mass ratio $>1 / 10$ that occurred between times $\left(t_{\mathrm{obs}}-t_{\mathrm{vis}}\right)$ and $t_{\mathrm{obs}}$. Systems of type Int-1 are identified empirically based on the following criteria:

1. They have $M_{*} \geqslant M_{\text {cut }}$ and show morphological distortions, which are similar to those seen in simulations of mergers of mass ratio $>1 / 10$, and remain visible over the visibility timescale $t_{\text {vis }}$. The distortions include arcs, shells, ripples, tidal tails, large tidal debris, highly asymmetric light distributions, double nuclei inside a common body, etc. The presence of such distortions is considered indicative of a past merger that occurred between times $\left(t_{\mathrm{obs}}-t_{\mathrm{vis}}\right)$ and $t_{\text {obs. }}$. We also make an extra test to verify that the distortions are caused by a past merger, rather than a present tidal interaction, by verifying that such systems do not have a distorted companion of similar spectrophotometric redshift within $40 \mathrm{kpc}$.

2. They appear as a single distorted system, rather than two individually recognizable galaxies, in the ACS images of PSF $\sim 0 ! 1$ (corresponding to $380 \mathrm{pc}$ at $z \sim 0.24$ and 

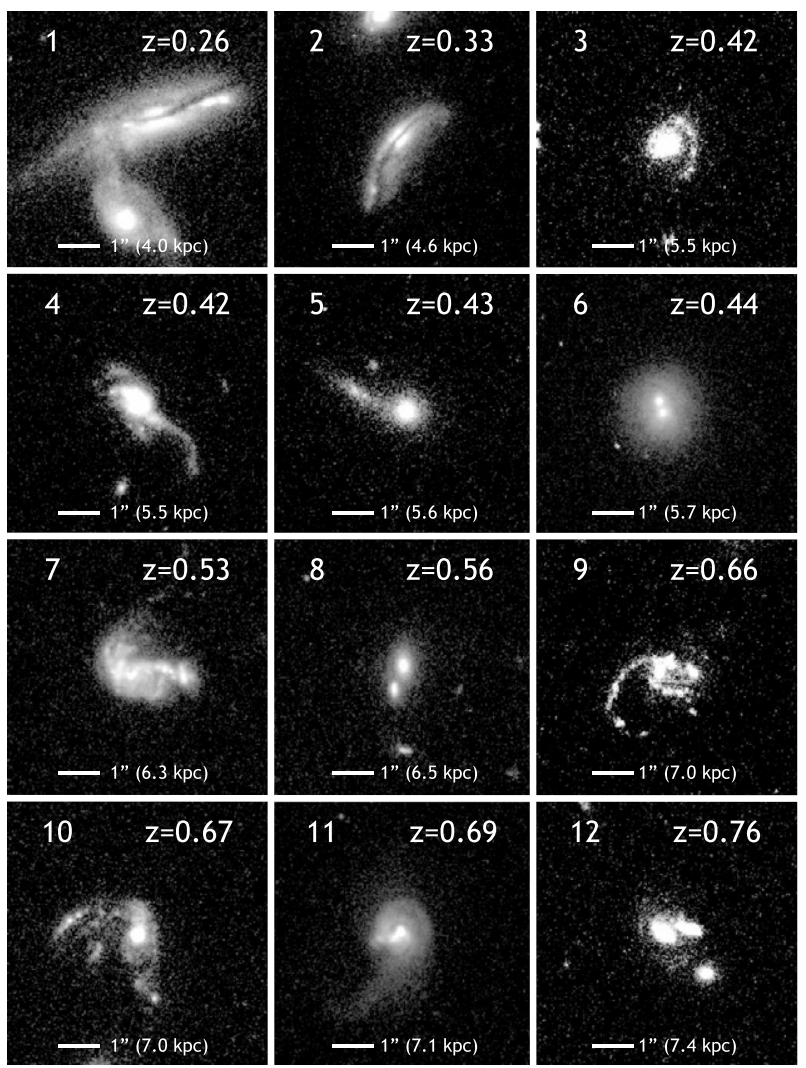

Figure 2. This montage show examples of mergers, namely systems that show evidence of having experienced a merger of mass ratio $>1 / 10$ within the last visibility timescale, as described in Section 3.2. The evidence includes morphological distortions similar to those seen in simulations of mergers of mass ratio $>1 / 10$, such as multiple nuclei (e.g., Case 6, 8); components (e.g., Case 12) connected by a bridge or common envelope; tidal tails and asymmetric features (e.g., Cases 3, 4, 5, 9, 11); and warped disks (e.g., Case 2). Systems classified as "Int-1" mergers primarily represent advanced mergers, which appear as single systems in ACS images (e.g., Cases 2, 3, 4, 5, 6, 7, 9, 10 11, and 12). Conversely, "Int-2" mergers (e.g., Case 1) primarily represent young mergers, which appear as VCPOGs in ACS images. The mergers can be divided into three groups: clear major mergers (Cases 1, 6, 12), clear minor mergers (Cases 2, 9), and ambiguous "major or minor mergers" (Cases 3, 4, 5, 7, 8, 10, 11).

$750 \mathrm{pc}$ at $z \sim 0.80$ ). This suggests the system is an advanced merger where the two progenitor galaxies have had time to coalesce into a single ACS system by time $t_{\mathrm{obs}}$.

Such advanced mergers have a single redshift (Wolf et al. 2004), stellar mass $M_{*}$ (Borch et al. 2006), and UV-based SFR (Bell et al. 2005, 2007) determined from the COMBO17 ground-based data of resolution $\sim 1^{\prime \prime} .5$. The lack of resolved COMBO-17 data for the individual progenitor galaxies that led to the remnant is not a problem because we are only concerned in the analysis with the stellar mass and SFR of the merger. Furthermore, the condition $M_{*} \geqslant M_{\text {cut }}$ is expected to apply to the merger, and not to the progenitors, in both model and observations.

The majority of the mergers we identify are of type Int-1. Examples in Figure 2 are Cases 2, 3, 4, 5, 6, 7, 9, 10 11, and 12.

For mergers of type Int-1 with a single redshift and stellar mass, the evidence for a merger of mass ratio $>1 / 10$ does not come from a measured stellar mass ratio $M 1 / M 2$, but instead is inferred from the presence of the aforementioned morphological distortions, which are seen in simulations of mass ratio $M 1 / M 2>1 / 10$
Without individual stellar masses $M 1$ and $M 2$, the further separation of these mergers into major and minor is not possible in every case, since the morphological disturbances induced depend not only on the mass ratio of the progenitors, but also on the orbital geometry (prograde or retrograde), the gas mass fraction, and structural parameters (e.g., Mihos \& Hernquist 1996; Struck 1997; Naab \& Burkert 2001; Mihos et al. 1995, di Matteo et al. 2007). Instead, we can only separate such mergers into three groups: clear major mergers, clear minor mergers, and ambiguous cases of "major or minor" merger as follows:

1. The class of clear major mergers includes systems that host fairly unique tell-tale morphological distortions characteristic of a major merger, such as two tails of equal lengths, two nuclei of similar luminosity, (e.g., Case 6 in Figure 2) and a train-wreck morphology (e.g., Case 12 in Figure 2).

2. The class of clear minor merger includes the merger systems where the outer disk (identified based on the presence of disk features, such as spiral arms and bars) has clearly survived the recent past merger. Examples include Case 2 of a warped disk in Figure 2. The reasoning behind classifying such remnants as a minor merger is that the outer disk of a galaxy survives a minor merger, but not a major merger. While major mergers of extremely gas-rich disks with low SF efficiency can lead to a remnant with an extended stellar disk (Robertson et al. 2004), such mergers are unlikely to be relevant for our study, which focuses on fairly massive systems (with stellar masses $\geqslant 1 \times 10^{9} M_{\odot}$ ) at $z<1$.

One further criterion is applied. When identifying minor mergers through the presence of a distorted surviving outer disk, we take care to check that the candidate is not in the early phases of a merger, which would destroy the disk in the near future, on a timescale $t_{\text {vis }}$. We do this by avoiding those galaxies, which have both a distorted outer disk and a close companion of similar redshift (within the photometric redshift accuracy) and of mass ratio $>1 / 4$.

3. The class of ambiguous 'major or minor' merger is assigned to systems hosting distortions, which could be due to both a major and a minor merger. Examples in Figure 2 are Cases $3,4,5,7,8,10$, and 11 .

\subsubsection{Mergers of type Int-2}

Mergers of type Int-2 primarily represent young mergers, which appear as very close pairs of overlapping galaxies (VCPOGs) in ACS images, have $M_{*} \geqslant M_{\text {cut }}$, and are likely associated with a merger of mass ratio $>1 / 10$ that occurred between times $\left(t_{\mathrm{obs}}-t_{\mathrm{vis}}\right)$ and $t_{\mathrm{obs}}$.

Systems of type Int-2 are identified empirically based on the following criterion: ACS images show two individually recognizable galaxies whose bodies overlap to form a common continuous envelope of light, and whose centers have a small separation $d<20 \mathrm{kpc}$. One or both of the galaxies often have morphological distortions. These properties suggest that the two progenitor galaxies have recently merged, at a time close to $t_{\mathrm{obs}}$, but have not yet coalesced into a single ACS system of type Int-1.

It is important to note that we are not concerned here with pairs of galaxies with a wide separation $d \geqslant t_{\mathrm{vis}} \times v$ (where $v$ is the relative speed between the two progenitor galaxies), because such systems represent potential future mergers that will occur in the next discrete time step of $\left(t_{\mathrm{obs}}+t_{\mathrm{vis}}\right)$. Instead, we are only interested in very close overlapping pairs of galaxies with separation $d \ll t_{\text {vis }} \times v$, which represent young mergers 
that have occurred at a past time close to $t_{\mathrm{obs}}$, and which will likely coalesce into a single ACS system well before the next discrete time step of $\left(t_{\mathrm{obs}}+t_{\mathrm{vis}}\right)$. For $t_{\mathrm{vis}}$ of $0.5 \mathrm{Gyr}$ and $v \sim 100 \mathrm{~km} \mathrm{~s}^{-1}, d \ll t_{\text {vis }} \times v$ translates to $d \ll 50 \mathrm{kpc}$. We thus only consider VCPOGs where the galaxies overlap and have a separation $d<20 \mathrm{kpc}$, which corresponds to $d<5$ ". 3 at $z \sim$ 0.24 and $d<2$. 8 at $z \sim 0.80$.

One caveat in handling systems of type Int-2 is that some of the VCPOGs may be chance projections rather than real gravitationally bound mergers. However, this uncertainty does not significantly affect our result as the vast majority (> 80\%) of the mergers in our study are of type Int- 1 rather than Int-2. Furthermore, the likelihood of chance projection is mitigated due to the fact that we are considering pairs of very small separation.

The COMBO-17 ground-based data of resolution $\sim 1$ 1".5 will resolve a fraction of the VCPOGs that make up systems of type Int-2. Thus, we divide the latter systems into sub-classes Int-2a and Int-2b:

1. Mergers of type Int-2a: These VCPOGs are not resolved by COMBO-17 data. Thus, only one stellar mass, redshift, and UV-based SFR are available for the pair. The lack of resolved COMBO-17 data for each galaxy in the pair is not a problem because we are only concerned with the mass and SFR of the progenitor. Furthermore, the condition $M_{*} \geqslant M_{\text {cut }}$ is expected to apply to the merger, and not to the progenitors, in both model and observations.

Since only one mass is available for the entire merged system, the evidence for a merger of mass ratio $>1 / 10$ in systems of type Int-2a does not come from a measured mass ratio, but instead is deduced from the presence of morphological distortions, which are seen in simulations of mass ratio $M 1 / M 2>1 / 10$. Essentially, the same approach described for systems of type Int-1 in Section 3.2.1 is used here.

2. Mergers of type Int-2b: These VCPOGs are resolved by COMBO-17 data such that stellar masses $M 1$ and $M 2$, as well as redshifts and UV-based SFRs, are available for both galaxies in the pair. An example is Case 1 in Figure 2). For systems of type Int-2b, the evidence for a merger of mass ratio $>1 / 10$ comes directly from the measured mass ratio $M 1 / M 2$. The SFR and mass of the merger is considered as the sum of that of its two progenitor galaxies

One caveat should be noted concerning the completeness of mergers of type Int-2b. Strictly speaking, the criterion $M_{*} \geqslant M_{\text {cut }}$ applies to the merger mass $(M 1+M 2)$ rather than to $M 1$ or $M 2$ individually. For $M_{\text {cut }} \sim 2.5 \times 10^{10} M_{\odot}$ counting all major mergers of type Int-2b with $1 / 4<M 1 / M 2 \leqslant 1 / 1$, requires our sample to be complete down to $1 \times 10^{10} M_{\odot}$ for 1:1 major mergers, and down to $8 \times 10^{9} M_{\odot}$ for 1:3 major mergers. Similarly, counting all minor mergers of type Int-2b with $1 / 10$ $<M 1 / M 2 \leqslant 1 / 4$ requires our sample to be complete down to $6 \times 10^{9} M_{\odot}$ for 1:4 minor mergers, and down to $2.5 \times 10^{9} M_{\odot}$ for 1:9 minor mergers. For the blue cloud, where we are complete down to $1.0 \times 10^{9} M_{\odot}$, our count of mergers of type Int-2b is complete, but there will inevitably be incompleteness on the red sequence, particularly among minor mergers. The impact of this incompleteness on our results is mitigated by the fact that most of the galaxies in our sample is on the blue cloud (see Figure 1) rather than on the red sequence, and the vast majority $(>80 \%)$ of the mergers in our study are of type Int- 1 rather than Int- 2 .

\subsection{Visual Classification of Non-Interacting Galaxies}

Systems that do not satisfy the criteria in Section 3.2 and show no evidence of a recent merger of mass ratio $>1 / 10$ are classified as non-interacting. These systems may harbor very subtle distortions, but none of the kind shown by the mergers of type "Int-1" and "Int-2". The non-interacting systems are divided into two sub-groups: non-interacting E-to-Sd and noninteracting Irr1. These are shown in Figure 3 and described below.

1. "Non-Interacting Irr1": It is important to note that even noninteracting galaxies have some inherent level of small-scale asymmetries in optical light due to SF activity. In the case of low mass galaxies, further asymmetries may also arise due to the low ratio of rotational to random velocities, as is commonly seen in Im and Sm. These internally triggered asymmetries due to SF in non-interacting galaxies differ in scale (few $100 \mathrm{pc}$ vs several kpc) and morphology from the externally triggered distortions typical of the "Int1 " class. We classify non-interacting galaxies with such internally triggered asymmetries as "Irr1" (see Figure 3). Such systems may get erroneously classified as mergers in automated asymmetry-based codes (see Section 4.2).

2. "Non-Interacting E-to-Sd": Galaxies are assigned the "E to Sd" class if they are fairly symmetric, have Hubble types in the range E-to-Sd, and are not associated with any overlapping or contact companion.

In this paper, we are primarily concerned about the differences between three groups: the mergers in class "Int", the non-interacting E-to-Sd galaxies, and the non-interacting Irr1 galaxies. The details of how E-to-Sd galaxies are further subdivided into individual Hubble types do not have any major impact on our main results. We nonetheless briefly describe this sub-classification as it is of interest to other studies and relevant for the test presented at the end of Section 4.1. We use conventional definitions (Binney \& Merrifield 1998) for individual Hubble types (E, S0, Sa, Sb-Sc, and Sd). We assign an elliptical (E) type if a galaxy exhibits a smooth featureless appearance, shows no disk signatures, such as a bar or spiral arms, and appears to be a pure spheroid. We assign an S0 class if a galaxy hosts a smooth central brightness condensation, surrounded by an outer component, which is relatively featureless (without spiral arms) and has a less steeply declining brightness profile. We assign $\mathrm{Sa}, \mathrm{Sb}-\mathrm{Sc}$, and $\mathrm{Sd}$ types using primarily visual estimates of the $B / D$ ratio, and secondarily the smoothness/clumpiness of the disk. At intermediate redshifts, where the faint smooth arms of Sa galaxies are not easily discernible, the distinction between E, S0, and Sa becomes blurred (see also Section 4.1). However, this ambiguity between Es, SOs and Sas is not a problem for the subsequent analyses in this paper, since galaxies are grouped together either as "E+S0+Sa" or "E-to-Sd".

The fraction of interacting systems (i.e., mergers), "noninteracting E-to-Sd" galaxies, and "non-interacting Irr1" galaxies is shown in Table 1 for the high-mass sample, and in Table 2 for the intermediate-mass sample. Further results and tests on the merger history from visual classes are presented in Sections 4.1 and 4.3.

\section{4. $C A S$}

We derived the concentration $C$, asymmetry $A$, and clumpiness $S$ (CAS) parameters by running the CAS code (Conselice 2003) on the F606W images. As is standard practice, the segmentation maps produced during the original source extraction 

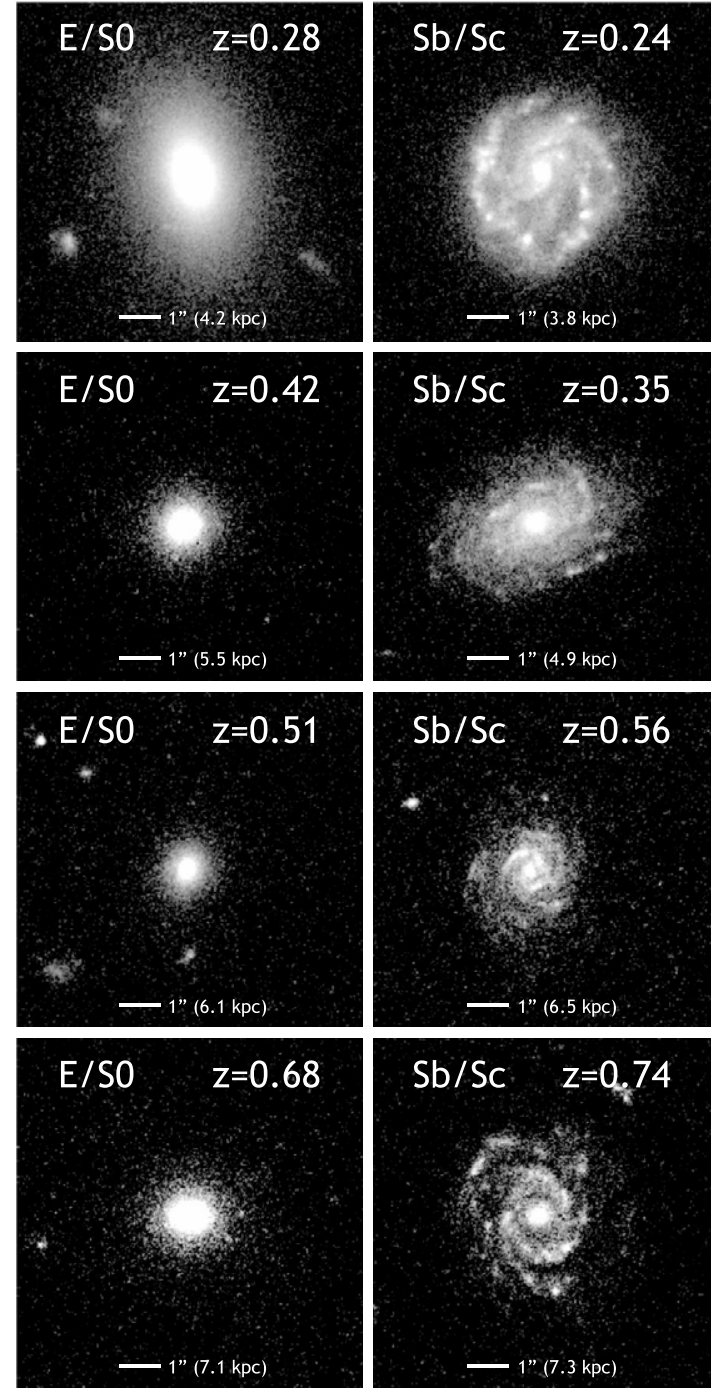
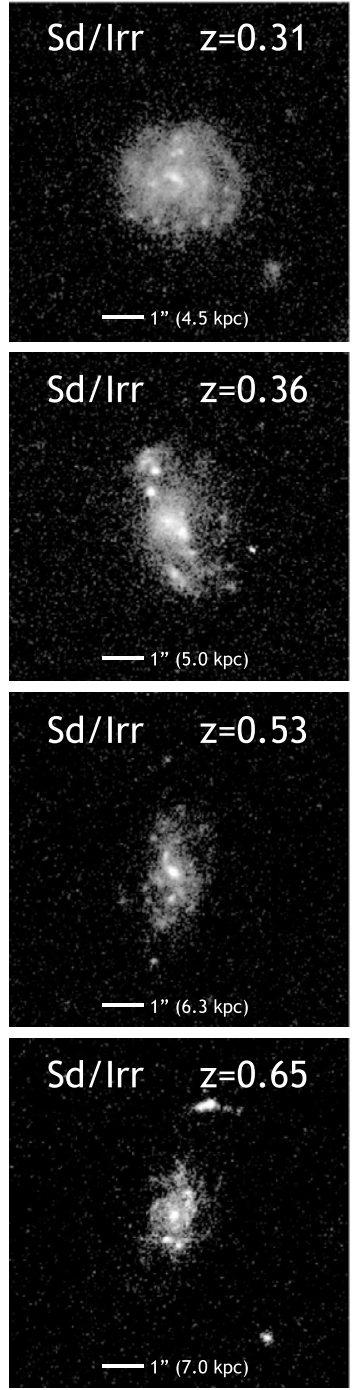

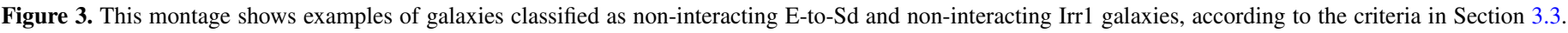
Within the broad class of non-interacting E-to-Sd, galaxies have Hubble types E, S0, Sa, Sb-Sc, and Sd, as shown in Figure 6.

(Caldwell et al. 2008) are used to mask neighbors on each ACS tile. The CAS code derives the asymmetry index $A$ (Conselice 2003 ) by rotating a galaxy image by $180^{\circ}$, subtracting the rotated image from the original image, summing the absolute intensities of the residuals, and normalizing the sum to the original galaxy flux. CAS improves the initial input center with the IRAF task "imcenter" and then performs a further refinement within a $3 \times 3$ grid, picking the center that minimizes $A$. The CAS concentration index $C$ (Bershady et al. 2000) is proportional to the logarithm of the ratio of the $80 \%$ to $20 \%$ curve of growth radii within 1.5 times the Petrosian inverted radius at $r(\eta=0.2)$, normalized by a logarithm

$$
C=5 \times \log \left(\mathrm{r}_{80 \%} / \mathrm{r}_{20 \%}\right) .
$$

The clumpiness index $S$ (Conselice 2003) is defined as the ratio of the amount of light contained in high-frequency structures to the total amount of light in the galaxy. In order to compute $S$, the CAS code first smooths the galaxy image with a filter of size equal to $1 / 6$ of the Petrosian radius to produce a lower resolution image whose high-frequency structure has been washed out. The latter image is then subtracted from the original image to produce a residual map that contains only the highfrequency components of the galaxy's stellar light. The flux of this residual light is then summed and divided by the sum of the original galaxy image flux to obtain a galaxy's clumpiness $(S)$ value. Tests on the interaction history from CAS are presented in Section 4.2.

It has been argued that the criterion $A>0.35$ and $A>S$ (henceforth referred to as the CAS merger criterion) captures galaxies that exhibit large asymmetries produced by major mergers (Conselice 2003). We will assess this in Section 4.2.

\section{RESULTS AND DISCUSSION}

\subsection{The Merger Fraction from Visual Classes}

Figure 4 compares the fraction $f$ of systems with evidence of a recent merger of mass ratio $>1 / 10$, based on visual classification by three classifiers (SJ, SM, KP). Results are shown for both the high-mass $\left(M \geqslant 2.5 \times 10^{10} M_{\odot}\right)$ sample $\mathrm{S} 1$ and the intermediate-mass $\left(M \geqslant 1 \times 10^{9} M_{\odot}\right)$ sample $\mathrm{S} 2$. On this figure, the plotted error bar for the merger fraction $f$ only includes the binomial term $[f(1-f) / N]^{1 / 2}$, for each bin of size $N$. The same trend is seen for all three classifiers and the maximum 
Table 3

Merger fraction in GEMS F606W $(V)$ and GOODS F850LP $(z)[N=855]$

\begin{tabular}{|c|c|c|c|c|c|c|}
\hline & & $\begin{array}{c}\text { GEMS } V \\
\text { Average }\end{array}$ & $\begin{array}{c}\text { GOODS } z \\
\text { Average }\end{array}$ & $\begin{array}{c}\text { GOODS } z \\
\text { SJ }\end{array}$ & $\begin{array}{c}\text { GOODS } z \\
\text { SM }\end{array}$ & $\begin{array}{c}\text { GOODS } z \\
\text { KP }\end{array}$ \\
\hline (1) & Fraction $f$ of merging galaxies & $0.046 \pm 0.007$ & $0.049 \pm 0.007$ & $0.051 \pm 0.007$ & $0.057 \pm 0.008$ & $0.038 \pm 0.006$ \\
\hline (2) & Ratio of $f$ in GOODS $z$ to GEMS $V$ & - & $1.06 \pm 0.22$ & $1.10 \pm 0.23$ & $1.20 \pm 0.25$ & $0.83 \pm 0.18$ \\
\hline
\end{tabular}

Notes. As a test for bandpass shift and surface brightness dimming, the table shows a comparison of the fraction of visual mergers based on GEMS F606W images and deeper redder GOODS F850LP images. The sample consists of the 855 intermediate-mass $\left(M \geqslant 1.0 \times 10^{9} M_{\odot}\right)$ galaxies at $z \sim 0.24-080$, which are common to both GEMS F606W and GOODS F850LP surveys. Columns are: (2) fraction $f$ of mergers in GEMS F606W. The error bar only includes the binomial term $[f(1-f) / N]^{1 / 2}$; (3) average fraction $f$ of mergers in GOODS F850LP based on results by classifiers (SJ,SM,KP), shown in Columns 4-6.

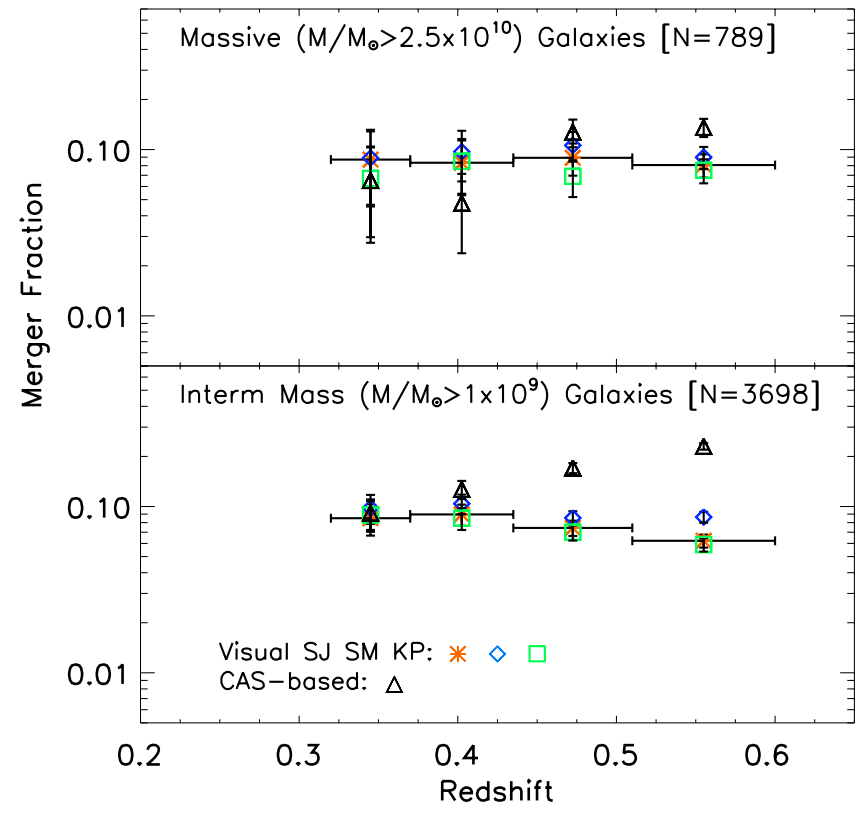

Figure 4. This figure compares the merger fraction $(f)$ based on visual classification by three classifiers (SJ, SM, KP), to merger fraction $\left(f_{\mathrm{CAS}}\right)$ that would be obtained using the CAS criterion $(A>0.35$ and $A>S)$. The results are shown for both high-mass $\left(M \geqslant 2.5 \times 10^{10} M_{\odot}\right.$; top panel) and intermediatemass $\left(M \geqslant 1 \times 10^{9} M_{\odot}\right.$; bottom panel) sample. The plotted error bar for $f$, at this stage, only includes the binomial term $[f(1-f) / N]^{1 / 2}$, for each bin of size $N$. The same trend is seen for all three classifiers and the maximum spread $\delta_{\mathrm{f}} / f$ in the four bins is $\sim 26 \%$. $f_{\text {CAS }}$ agrees within a factor of 2 with the visually based $f$ merger fraction for high-mass galaxies. However, for intermediate-mass galaxies, CAS can overestimate the merger fraction at $z>0.5$ by a factor $\sim 3$, as it picks up a significant number of non-interacting dusty, star-forming galaxies (see Section 4.2).

(A color version of this figure is available in the online journal.)

spread $\delta_{\mathrm{f}} / f$ in the four bins is $15 \%, 17 \%, 26 \%$, and $26 \%$, respectively. In subsequent analyses, we adopt a conservative error bar on $f$ that includes in quadrature both the binomial term and a dispersion of $26 \%$ to capture the inherent subjectivity in the visual classification.

Another key test is to assess the impact of redshift-dependent systematic effects, such as bandpass shifting. When using the F606W filter whose pivot wavelength is $\sim 5915 \AA$, the rest frame wavelength $\left(\lambda_{\text {rest }}\right)$ corresponds to the rest-frame optical at the mean redshift of the first three bins, but shifts to the rest-frame violet/near-UV (3700 $\AA-3290 \AA)$ in the last bin $(z \sim 0.6-0.8)$. Galaxies tend to look slightly more asymmetric at near-UV wavelengths due to the prominence of young stars. In order to quantitatively test the impact of bandpass shift on our visual classes, we use the redder F850LP images from the GOODS survey, which overlaps with the central $20 \%$ of the GEMS survey area. The F850LP filter has a pivot wavelength of $9103 \AA$ and traces the rest-frame optical $(7340 \AA-5057 \AA)$ in all four redshift bins out to $z \sim 0.8$. The F850LP images also have 5 times longer exposures than the GEMS F850LP and F606W images. Figure 5 shows GEMS F606W and GOODS F850LP images of typical disturbed and normal galaxies in the last two redshift bins $(z \sim$ 0.47-0.8).

While the GOODS images have higher $\mathrm{S} / \mathrm{N}$, and trace redder older stars, they do not reveal dramatically different morphologies from those in the GEMS F606W images (Figure 5). Furthermore, the 855 intermediate-mass $(M \geqslant$ $\left.1 \times 10^{9} M_{\odot}\right)$ galaxies in the GEMS/GOODS overlap area, were classified using both GOODS F850LP and GEMS F606W images by the three classifiers. We find that the ratio of $\left(f_{\mathrm{GEMS}} / f_{\mathrm{GOODS}}\right)$ ranges from 0.8 to 1.2 across the three classifiers (Table 3 ), where $f_{\text {GEMS }}$ and $f_{\text {GOODS }}$ are the fraction of merging systems based on the GEMS F606W and GOODS F850LP images, respectively. The mean $f$ changes by only $6 \%$ (Table 3). In effect, over $85 \%$ of the systems classified as mergers ("Int") in the GEMS F606W images retain the same visual class in the GOODS F850LP. Among the remaining objects, some classified as non-interacting in GEMS F606W get reclassified as disturbed in GOODS F850LP, and vice versa. The fact that $f$ does not change by a large amount between GEMS F606W and GOODS F850LP is not surprising, since the restframe wavelength of GEMS F606W in the last bin shifts only to the violet/near-UV, rather than to the far-UV, where morphological changes are more dramatic. We conclude that our results are not highly impacted by bandpass shifting, and any effect is accounted for by our error bars of $>26 \%$ in $f$.

Another redshift-dependent systematic effect is surface brightness dimming at the rate of $(1+z)^{-4}$ (e.g., Barden et al. 2008). This leads to surface brightness dimming by a factor of $1.0-2.5 \mathrm{mag}$ over the redshift range $0.24-0.80$. This is mitigated in part by two factors: galaxies are on average $1.0 \mathrm{mag}$ brighter in surface brightness by $z \sim 0.8$ (e.g., Barden et al. 2005), and the average SFR rises by a factor of $\sim 4$ out to $z \sim 0.8$ (e.g., see Section 4.6). Two approaches can be adopted to assess the impact of surface brightness dimming. The first is to artificially redshift disturbed galaxies in the lowest redshift bin $(z \sim 0.24)$ out to $z \sim 0.8$, either assuming passive evolution or adding in a $\sim 1$ mag of brightening in surface brightness. However, this approach suffers from the limitation that it implicitly assumes that galaxies at $z \sim 0.8$ are similar to those at $z \sim 0.24$ and evolve passively with time. A better approach, which does not make such assumptions, is to repeat the analysis and visual classification using deeper images of the galaxies and assess the resulting change in visual classes. The above-described test performed using the deep GOODS F850LP image (Figure 5) is an example of such a test, and indicates that the eye-ball morphologies do not change within the error bars of $>26 \%$ in $f$. We note however that quantitative CAS parameters can change with the deeper GOODS images (e.g., Conselice et al. 2008). 

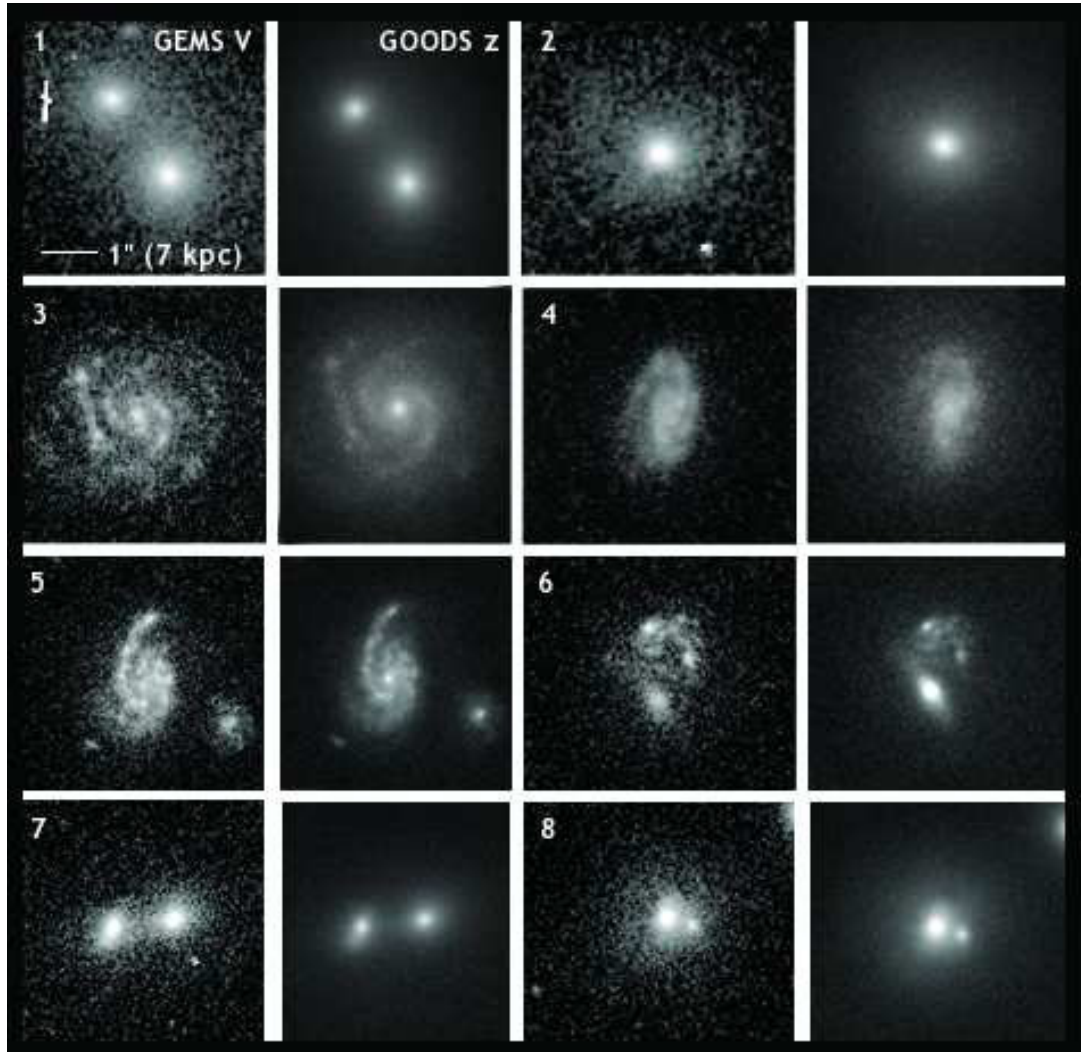

Figure 5. This montage illustrates a test for bandpass shift and surface brightness dimming. It compares the bluer shallower GEMS F606W images ( $V$ band; pivot $\lambda \sim 5915 \AA)$ and deeper redder GOODS F850LP $(z$ band; pivot $\lambda \sim 9103 \AA)$ images of typical interacting and non-interacting galaxies in the last redshift bin $(z \sim$ $0.60-0.80$ ), where bandpass shift and surface brightness dimming are expected to be most severe. In this redshift bin, the rest-frame wavelength traced by the GEMS images shift from optical to violet/near-UV (3700 $\AA-3290 \AA$ ). However, while the GOODS images have higher S/N, and trace redder older stars, they do not reveal dramatically different morphologies from those in the GEMS F606W images. Cases 1, 5, 6, 7, and 8 are interacting systems. A statistical analysis is shown in Table 3 .

Finally, as an extra test, we checked the distribution of Sérsic indices $n$ for single-component Sérsic fits (Barden et al. 2005) for the visual classes of the sample S2 of intermediate-mass $M \geqslant 1 \times 10^{9} M_{\odot}$ galaxies (Figure 6). Non-Interacting diskdominated systems are expected to have $n<2.5$, while massive ellipticals and bulge-dominated systems typically have higher Sérsic indices. We indeed find that over $85 \%$ of the systems visually classified as $\mathrm{Sb}-\mathrm{Sd}$ and $\operatorname{Irr} 1$ have $n<2.5$ in the intermediate-mass $\left(M \geqslant 1 \times 10^{9} M_{\odot}\right)$ sample. Furthermore, as expected, the vast majority of the systems typed as $\mathrm{Sa}$ have $n<4$. However, the systems typed as E and S0 span a broad range in $n$ : most of them have $n>3$, but there is a tail of lower $n$ values. This is not surprising given the previously described difficulties (Section 3.3) in separating E, S0, and Sa galaxies at intermediate redshifts. However, this ambiguity between E, S0, and Sa systems is not a problem for the subsequent analyses in this paper, since galaxies are grouped together either as "E+S0+Sa" or "E-to-Sd". In fact, as stressed in Section 3.3, the main results presented in this paper depend only on the differences between three groups: mergers ("Int"), noninteracting E to Sd galaxies, and non-interacting Irr1 galaxies.

\subsection{The Merger Fraction from $C A S$}

It has been argued that the CAS merger criterion $(A>$ 0.35 and $A>S$ ) captures systems that exhibit large asymmetries produced by major mergers (Conselice 2003). This criterion is based on calibrations of the CAS system at optical rest-frame wavelengths $\left(\lambda_{\text {rest }}>4500 \AA\right)$. However, there are several caveats. (a) The CAS criterion $(A>0.35$ and $A>S)$ will miss out interacting galaxies where the morphological distortions contribute to less than $35 \%$ of the total galaxy flux. (b) Calibrations of $A$ with $N$-body simulations (Conselice 2006) shows that during major mergers with mass ratios 1:1-1:3, the asymmetry oscillates with time. Typically, it exceeds 0.35 for $\sim 0.2$ Gyr in the early phases when the galaxies start to interact, falls to low values as the galaxies separate, rises for $\sim 0.2 \mathrm{Gyr}$ as they approach again for the final merger, and eventually tapers down as the final remnant relaxes. On average, the $A>0.35$ criterion is only satisfied for one third of the merger timescale in these $N$-body simulations. For minor mergers of mass ratios 1:5 and below, the asymmetries are too low to satisfy $A>0.35$. (c) To complicate matters, automated asymmetry parameters can also capture non-interacting galaxies whose visible light shows small-scale asymmetries due to SF (e.g., Miller et al. 2008; Lotz et al. 2008).

Visual tests that verify how well the CAS criterion $(A>$ 0.35 and $A>S$ ) works at intermediate redshifts have been performed using spot checks and small-to-moderate samples (e.g., Mobasher et al. 2004; Conselice 2003; Conselice et al. 2003 , 2005). However, what has been missing to date is a quantitative estimate, based on a large sample of galaxies, of the recovery fraction of CAS (i.e., the fraction of visually classified mergers that the CAS criterion picks up), and the contamination level of CAS (i.e., the fraction of visually classified noninteracting galaxies that the CAS criterion picks up). Both the recovery fraction and contamination level might be expected to depend on the rest-frame wavelength used, the mass and SFR 


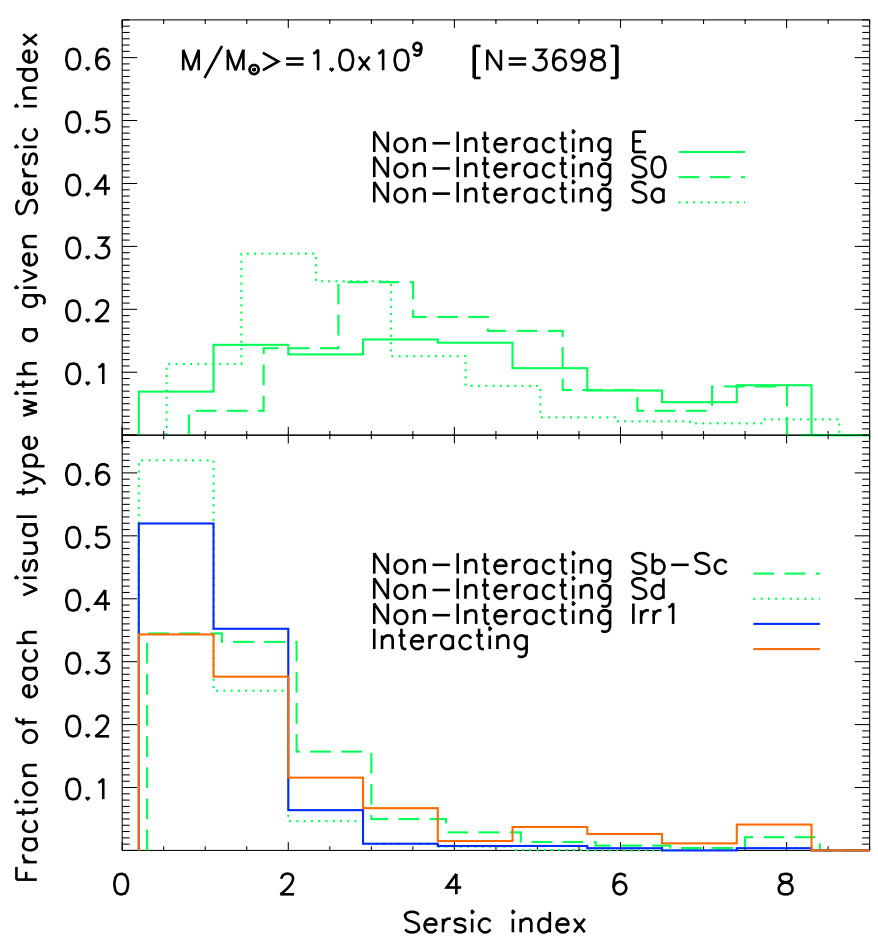

Figure 6. For intermediate-mass $\left(M \geqslant 1 \times 10^{9} M_{\odot}\right)$ galaxies, the distribution of Sérsic index $n$ from single-component Sérsic fits is plotted for mergers, non-interacting Irr1, and non-interacting E-to-Sd systems. The latter class is further subdivided as E, S0, Sa, Sb-Sc, and Sd. The majority of systems visually classified as non-interacting Sb-Sc, $\mathrm{Sd}$, and Irr1 have $n<2.5$, as expected for disk-dominated systems. Most of the systems visually typed as Sa have $n<4$. Those galaxies typed as E and S0 primarily have $n>3$, but some have low $n$, reflecting the inherent difficulty in separating $\mathrm{E}, \mathrm{S} 0$, and $\mathrm{Sa}$ at intermediate redshifts.

(A color version of this figure is available in the online journal.)

of the galaxies, etc. In this paper, we perform one of the most extensive comparisons to date, at intermediate redshifts $(z \sim$ $0.24-0.80$ ), between CAS-based and visual classification results for both high-mass $\left(M \geqslant 2.5 \times 10^{10} M_{\odot}\right)$ and intermediate-mass $\left(M \geqslant 1 \times 10^{9} M_{\odot}\right)$ galaxies. We assess the effectiveness of the CAS merger criterion $(A>0.35$ and $A>S$ ) over this interval, where the rest-frame wavelength $\lambda_{\text {rest }}$ varies from $4770 \AA$-3286 $\AA$. We note that the rest-frame wavelength range here extends to somewhat bluer wavelengths than the range $\left(\lambda_{\text {rest }}>4500 \AA\right)$ over which the CAS system was calibrated.

Figure 4 compares the merger fractions that would be obtained using the CAS criterion $\left(f_{\mathrm{CAS}}\right)$, as opposed to visual classification $(f)$. For the high-mass $\left(M \geqslant 2.5 \times 10^{10} M_{\odot}\right)$ galaxies, visually based and CAS-based merger fractions agree within a factor of 2 , with $f$ being higher than $f_{\text {CAS }}$ at $z<0.5$, and being lower at $z>0.5$ (top panel of Figure 4). However, for the intermediate-mass $\left(M \geqslant 1 \times 10^{9} M_{\odot}\right)$ galaxies (lower panel of Figure 4), at $z>0.5$ the CAS-based merger fraction can be systematically higher by a factor $\sim 3$ than the visually based $f$. The reason for this discrepancy, as we show below, is that at bluer rest-frame wavelengths (i.e., higher redshifts), the CAS criterion picks up a significant number of non-interacting dusty, star-forming galaxies.

Figure 7 plots the CAS asymmetry $A$ and clumpiness $S$ parameter for galaxies in the four redshift bins covering the interval $z \sim 0.24-0.80$. Galaxies satisfying the CAS criterion $(A>0.35$ and $A>S$ ) lie in the upper left hand corner. One can see that while the CAS criterion captures a fair fraction of the

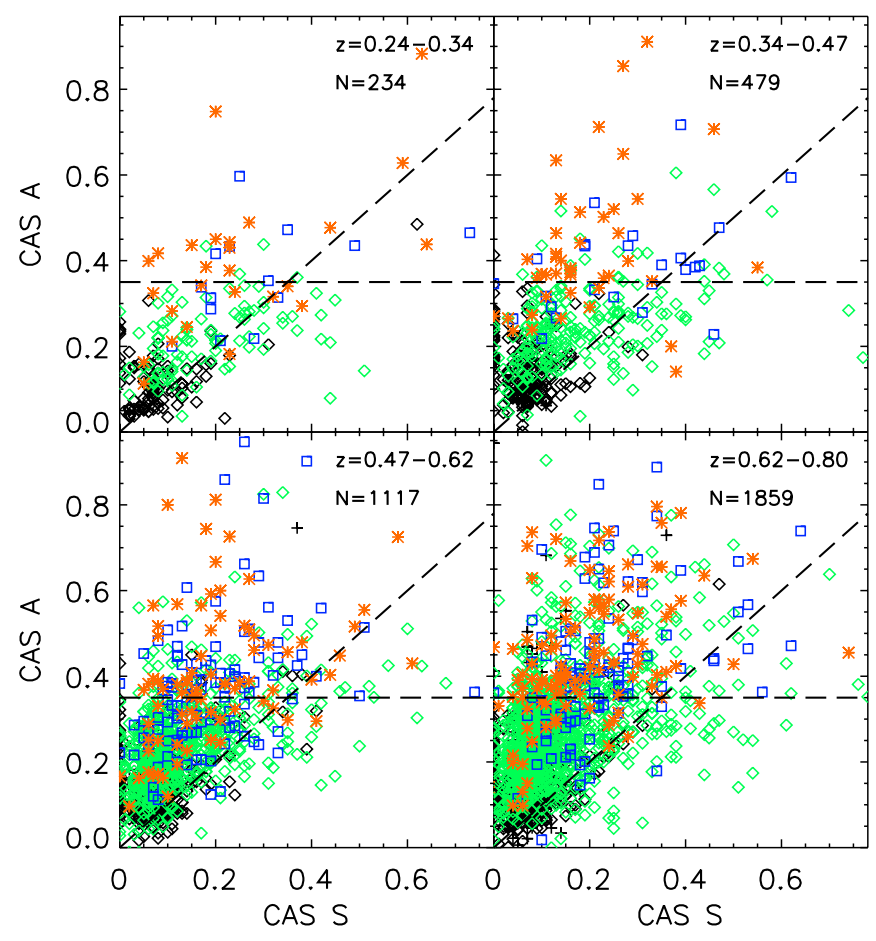

Figure 7. CAS asymmetry $A$ and clumpiness $S$ parameters are plotted for intermediate-mass $\left(M \geqslant 1 \times 10^{9} M_{\odot}\right)$ galaxies in the four redshift bins of Figure 1, using the same color coding. Galaxies satisfying the CAS criterion $(A>0.35$ and $A>S$ ) lie in the upper left hand corner, bracketed by the $A=$ $S$ diagonal line and the $A=0.35$ horizontal line. The CAS criterion captures a fair fraction of the galaxy mergers, but it also picks up "contaminants" in the form of non-interacting galaxies. This is further illustrated in Figure 8.

(A color version of this figure is available in the online journal.)

mergers (coded as orange stars), it also picks up a large number of non-interacting galaxies.

We define the recovery fraction $\left(F_{\mathrm{CAS}-\text { merger }}\right)$ of CAS as the fraction of visually classified mergers ("Int"), which are picked up by the CAS criterion $(A>0.35$ and $A>S$ ). For the high-mass sample $\left(M \geqslant 2.5 \times 10^{10} M_{\odot}\right)$ sample, the recovery fractions in the four redshift bins are $50 \%(2 / 4), 14 \%(1 / 7)$, $42 \%(8 / 19)$, and 56\% (20/36) respectively, with low number statistics dominating the first two bins. For the intermediatemass $\left(M \geqslant 1 \times 10^{9} M_{\odot}\right)$ sample, the recovery fractions in the four redshift bins are 50\% (13/26), 69\% (30/43), 59\% (49/83), and $73 \%(85 / / 116)$, respectively, as illustrated in the top panel of Figure 8 . We inspected the visually classified mergers missed out by the CAS criterion $(A>0.35$ and $A>S$ ) and show typical cases in the top panel of Figure 9. The missed cases include galaxies where tidal or accretion features in the main disk of a galaxy contribute less than $35 \%$ of the total light (e.g., Case 3 in Figure 9); galaxies with close double nuclei (e.g., Case 2 in Figure 9) where CAS might refine the center to be between the two nuclei, thereby leading to a low $A<0.35$; and pairs of fairly symmetric galaxies whose members have similar redshifts within the spectrophotometric error, appear connected via weak tidal features, and have a stellar mass ratio $M 1 / M 2>1 / 10$ (e.g., Case 1 in Figure 9 where $M 1 / M 2 \sim 0.25$ ).

We define the contamination fraction of CAS as the fraction of those systems which satisfy the CAS criterion $(A>0.35$ and $A>S$ ) and are therefore considered as likely major mergers by CAS, but yet are visually classified as non-interacting. For the high-mass sample $\left(M \geqslant 2.5 \times 10^{10} M_{\odot}\right)$ sample, the CAS contamination fractions in the four redshift bins are $34 \%$, 


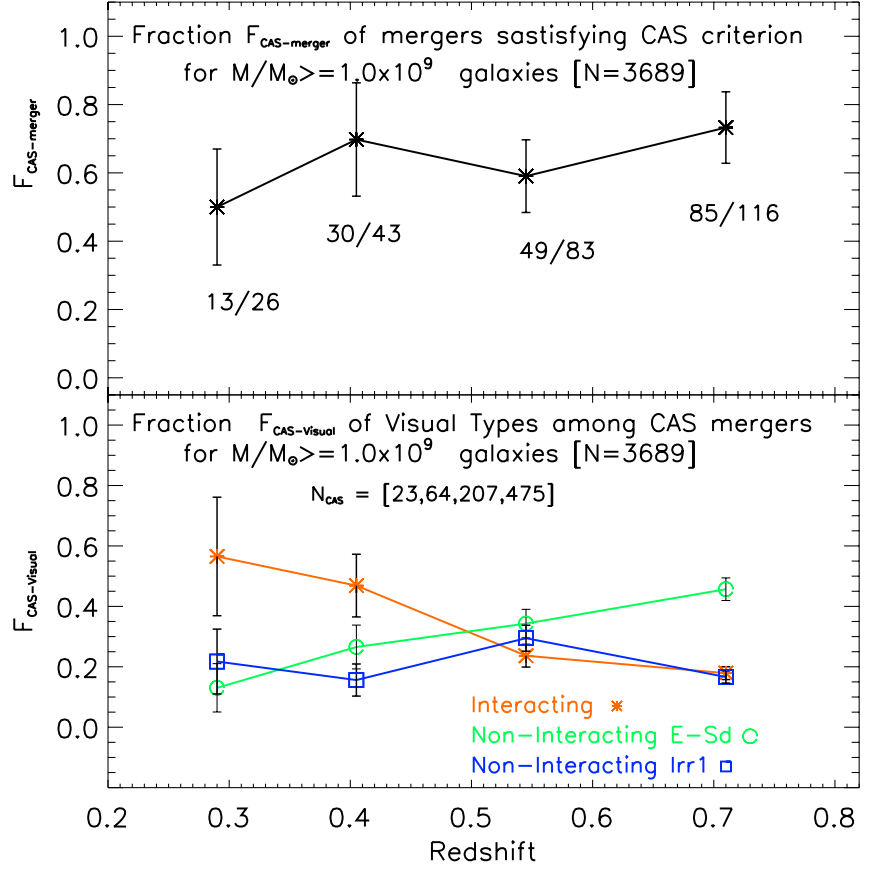

Figure 8. For galaxies with $M \geqslant 1.0 \times 10^{9} M_{\odot}$, the top panel shows that

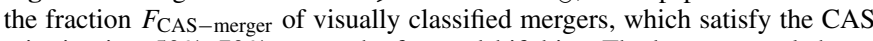
criterion is $\sim 50 \%-70 \%$ across the four redshift bins. The bottom panel shows the degree to which non-interacting galaxies contaminate the systems picked up by CAS. $N_{\text {CAS }}$ represents the total number of galaxies, which satisfy the CAS criterion and are considered as "CAS mergers" across the four redshift bins. The fraction $F_{\mathrm{CAS}-\text { visual }}$ of different visual types among these "CAS mergers" is plotted on the y-axis. At $z>0.5$, the vast majority (44\%-80\%) of the systems considered as mergers by the CAS criterion turn out to be non-interacting [E-Sd and Irr1] systems.

(A color version of this figure is available in the online journal.)

$75 \%, 72 \%$, and $67 \%$ respectively, with low number statistics dominating the first two bins. For the intermediate-mass $(M \geqslant$ $\left.1 \times 10^{9} M_{\odot}\right)$ sample, the corresponding CAS contamination fractions are $44 \%, 53 \%, 76 \%$, and $82 \%$ respectively, as shown in the lower panel of Figure 8 . On the latter figure, $N_{\text {CAS }}$ represents the total number of galaxies satisfying the CAS criterion $(A>0.35$ and $A>S$ ) in the four redshift bins. Plotted on the y-axis is the fraction $F_{\mathrm{CAS} \text {-visual }}$ of different visual types (mergers, non-interacting E-Sd, and non-interacting Irr1) among these "CAS mergers." Across the four redshift bins, the non-interacting E-Sd and Irr1 make up 44\%, 53\%, 76\%, and $82 \%$ of the CAS systems. Typical cases are shown in the lower panel of Figure 9. They include non-interacting actively starforming systems where SF induces small-scale asymmetries in the optical blue light (e.g., Cases 4 and 6 in Figure 9); systems where $A$ is high due to the absence of a clearly defined center (e.g., Case 8 in Figure 9) or due to the center being blocked by dust (e.g., Cases 4 and 9 in Figure 9); and compact or edge-on systems where the light profile is steep such that small centering inaccuracies can lead to large $A$ (e.g., Case 9 in Figure 9).

In summary, we find that the CAS-based merger fraction agrees within a factor of 2 with visually based one for highmass $\left(M \geqslant 2.5 \times 10^{10} M_{\odot}\right)$ galaxies, but can overestimate the merger fraction at $z>0.5$ by a factor $\sim 3$ for intermediatemass $\left(M \geqslant 1 \times 10^{9} M_{\odot}\right)$ galaxies. For the latter mass range, the systems counting toward $f_{\text {CAS }}$ are a mixed bag: the CAS criterion misses about half of the visually classified mergers, but picks up a dominant number of non-interacting dusty, star-forming galaxies. We thus conclude that the CAS merger criterion is ill-suited for use on HST V-band images at $z>0.5$, where the rest frame wavelength falls below $\lambda<4000 \AA$, particularly in the case of intermediate-mass galaxies with significant SF, gas, and dust. Modified CAS criteria in the near-UV based on morphological k-corrections (Taylor-Mager et al. 2007) might alleviate this problem.

\subsection{Interaction History of Massive and Intermediate-Mass Galaxies}

Based on the tests in Sections 4.1 and 4.2, we decided to adopt the mean merger fraction $f$ based on visual classes for our two samples of interest. For the high-mass $(M \geqslant$ $\left.2.5 \times 10^{10} M_{\odot}\right)$ sample, which is complete on both the blue cloud and red sequence (Section 2), the results are shown on in Table 1. The error bar shown on the merger fraction $f$ in both tables now includes the sum in quadrature of a binomial term $[f(1-f) / N]^{1 / 2}$ for each bin of size $N$, along with a fractional error of $\pm 26 \%$ to capture the dispersion between classifiers, and uncertainties due to bandpass shifting and surface brightness dimming.

From Table 1 and Figure 4, it can be seen that the merger fraction $f$ among high-mass $\left(M \geqslant 2.5 \times 10^{10} M_{\odot}\right)$ galaxies does not show strong evolution over lookback times of 3-7 Gyr, ranging from $9 \% \pm 5 \%$ at $z \sim 0.24-0.34$, to $8 \% \pm 2 \%$ at $z \sim 0.60-0.80$, as averaged over every Gyr bin. As discussed in Section 3.2, the merger fraction $f$ refers to systems with evidence of a recent merger of mass ratio $>1 / 10$.

As outlined in Section 3.2, these mergers were further subdivided among three classes: clear major merger, clear minor merger, and ambiguous "major or minor merger" cases. The first two classes are used to set lower limits on the major and minor merger fraction. The lower limit on the major $(M 1 / M 2>1 / 4)$ merger fraction, determined in this way, ranges from $1.1 \%$ to $3.5 \%$ over $z \sim 0.24-0.80$. The corresponding lower limit on the minor $(1 / 10 \leqslant M 1 / M 2<1 / 4)$ merger fraction ranges from $3.6 \%$ to $7.5 \%$. To our knowledge, this is the first, albeit approximate, empirical estimate of the frequency of minor mergers over the last 7 Gyr. The ambiguous cases of "major or minor merger" make up a fraction between $1.2 \%$ and $2.0 \%$.

When converting the observed fraction $f$ of galaxy mergers into a merger rate $R$, we must bear in mind that in any observational survey of galaxies, mergers can only be recognized for a finite time $t_{\mathrm{vis}}$, which is the timescale over which a merging galaxy will appear morphologically distorted. This timescale depends on the mass ratio of the merger as well as the gas fraction of the progenitors: $t_{\mathrm{vis}} \sim 0.5-0.8$ for gas-rich galaxies, and $t_{\mathrm{vis}} \sim 0.2-0.4$ Gyr for gas-poor galaxies (T.J. Cox 2008, private communication). This timescale will also depend on many observational factors such as the method used to identify mergers (e.g., visual classification vs. CAS or other statistical methods) and the depth of the imaging used. We assume a representative value of $t_{\mathrm{vis}}=0.5 \mathrm{Gyr}$ here, but we must keep in mind that there are at least factors of two uncertainty in this number. The merger rate $R$ is given by

$$
R=\frac{n f}{t_{\mathrm{vis}}}
$$

where $n$ is the comoving number density of galaxies above a certain mass limit in the redshift bin.

For the sample of high-mass $\left(M \geqslant 2.5 \times 10^{10} M_{\odot}\right)$ galaxies, our measured merger fraction $f$ and assumed value of $t_{\text {vis }} \sim 0.5$ Gyr lead to a corresponding merger rate $R$ of a few 


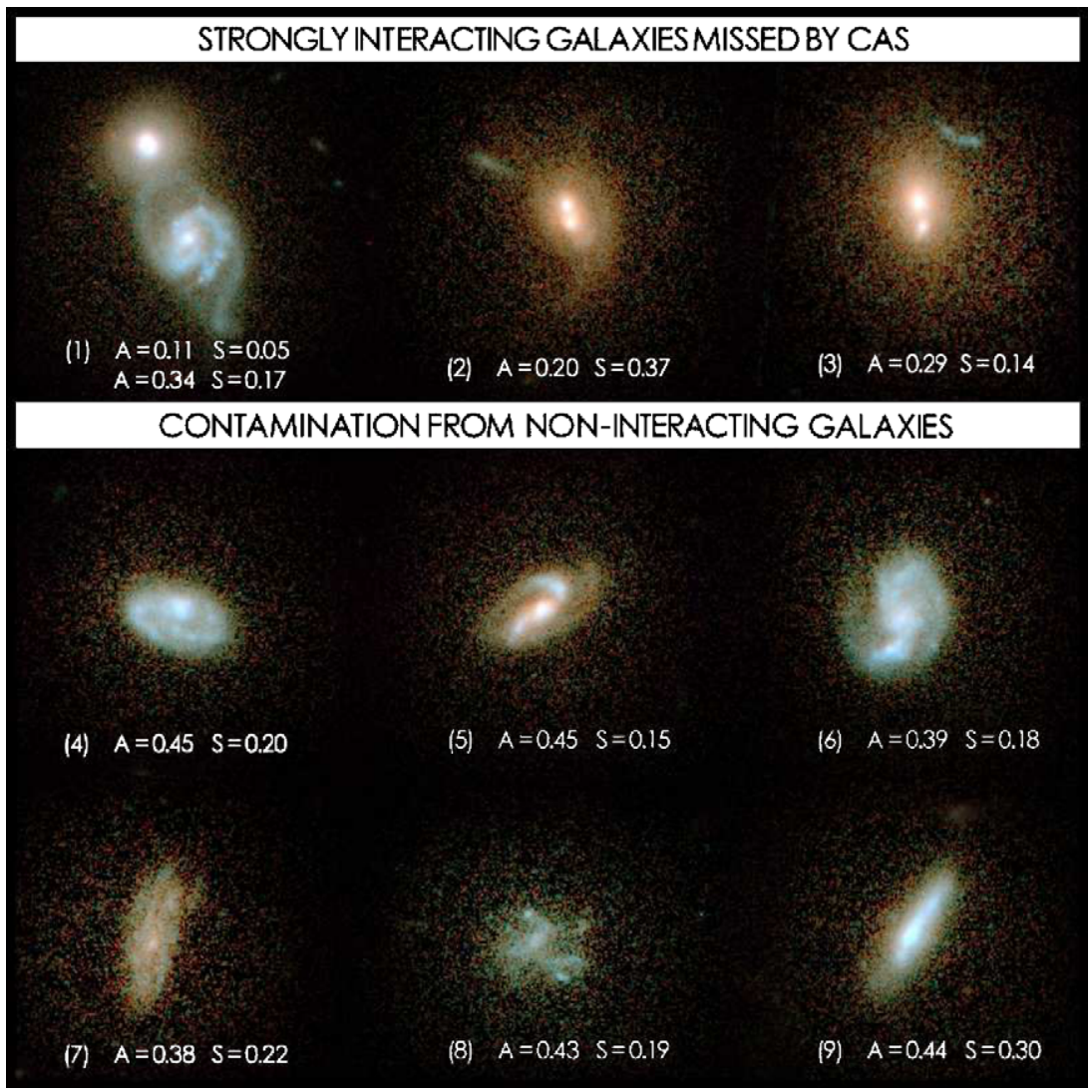

Figure 9. Montage shows typical systems where the CAS criterion $(A>0.35$ and $A>S)$ fails (see Section 4.2 for details). Cases $1-3$ are systems, which are visually classified as mergers, but are missed by the CAS criterion. They include systems with tidal debris (e.g., Case 3) that may contribute less than $35 \%$ of the total light; systems with close double nuclei (e.g., Case 2 ) where CAS might refine the center to be between the two nuclei, thereby leading to a low $A<0.35$; and pairs of fairly symmetric galaxies whose members have similar redshifts within the spectrophotometric error, appear connected via weak tidal features, and have a stellar mass ratio $M 1 / M 2>1 / 10$ (e.g., Case 1 where $M 1 / M 2 \sim 0.25$ ). Conversely, Cases $4-9$ are systems, which are visually classified as non-interacting galaxies, but are picked by the CAS criterion. They include non-interacting, actively star-forming systems with small-scale asymmetries in the optical blue light (Cases 4 and 6); systems where $A$ is high due to the absence of a clear center (Case 8 ) or due to the center being blocked by dust (Case 4, 9); edge-on systems and compact systems, where the light profile is steep such that small centering inaccuracies lead to large $A$ (Case 9).

(A color version of this figure is available in the online journal.)

$\times 10^{-4}$ galaxies $\mathrm{Gyr}^{-1} \mathrm{Mpc}^{-3}$. Assuming a visibility timescale of $\sim 0.5 \mathrm{Gyr}$, it follows that on average, over $T_{\mathrm{b}} \sim 3-7 \mathrm{Gyr}$, $\sim 68 \%$ of high-mass systems have undergone a merger of mass ratio $>1 / 10$. Of these, we estimate that $\sim 16 \%, 45 \%$, and $7 \%$ correspond respectively to clear major mergers, clear minor mergers, and ambiguous cases of "major or minor" mergers.

At intermediate masses $\left(M \geqslant 1 \times 10^{9} M_{\odot}\right)$ where we are only complete in mass for the blue cloud (Section 2), we consider $f$ to be meaningful only for the intermediate-mass blue-cloud sample. Results for this sample are shown in the lower part of Table 2. The fraction of blue-cloud galaxies having undergone recent mergers of mass ratio $>1 / 10$ ranges from $7 \% \pm 2 \%$ to $15 \% \pm 5 \%$ over $z \sim 0.24-0.80$. The corresponding merger rate $R$ ranges from $8 \times 10^{-4}$ to $1 \times 10^{-3}$ galaxies $\mathrm{Gyr}^{-1} \mathrm{Mpc}^{-3}$. For an assumed visibility time of $\sim 0.5 \mathrm{Gyr}$, we estimate that on average, over $T_{\mathrm{b}} \sim 3-7 \mathrm{Gyr}, 84 \%$ of intermediate-mass bluecloud galaxies have undergone a merger of mass ratio $>1 / 10$, with $\sim 5 \%, 22 \%$, and $57 \%$ corresponding respectively to clear major mergers, clear minor mergers, and ambiguous cases of "major or minor" mergers.

\subsection{Comparison with Other Studies}

When comparing our observed merger fraction $f$ in the highmass $\left(M \geqslant 2.5 \times 10^{10} M_{\odot}\right)$ sample over $z \sim 0.24-0.80$ with published studies, several caveats must be borne in mind. Many studies have small samples and large error bars at $z<0.8$ (e.g., Conselice 2003; Figure 10). Others focus on bright galaxies and luminosity-selected samples (e.g., Lotz et al. 2008; Cassata et al. 2005) rather than stellar mass selected sample, because the data to derive stellar masses were unavailable. Different studies target different systems, ranging from morphologically distorted systems to close pairs with separation $d \sim 5-40 \mathrm{kpc}$. Finally, many studies focus only on major mergers, while the interacting galaxies identified in our study are candidates for a merger of mass ratio $>1 / 10$ (Section 3.2), and include both minor and major mergers. Nonetheless, we attempt approximate comparisons.

Figure 10 shows the merger fraction based primarily on morphologically distorted galaxies (filled circles), as well as the close pair fraction (open squares), as a function of redshift. The Lotz et al. (2008) study shows the fraction $f_{\text {Gini }}$ of morphologically disturbed systems based on Gini-M20 parameters among $M_{\mathrm{B}}<-20.5$ and $L_{\mathrm{B}}>0.4 L_{*}$ galaxies in the Extended Groth Strip. This study does not present any results for a high-mass sample, and thus we effectively are comparing their bright galaxies to our high-mass galaxies. Over $z \sim 0.2-0.80$, our results are in very good agreement, within a factor of less than 2 , with $f_{\text {Gini }}$. The CAS-based results from Conselice (2003) are derived from a small sample in the Hubble Deep Field and have error bars that 


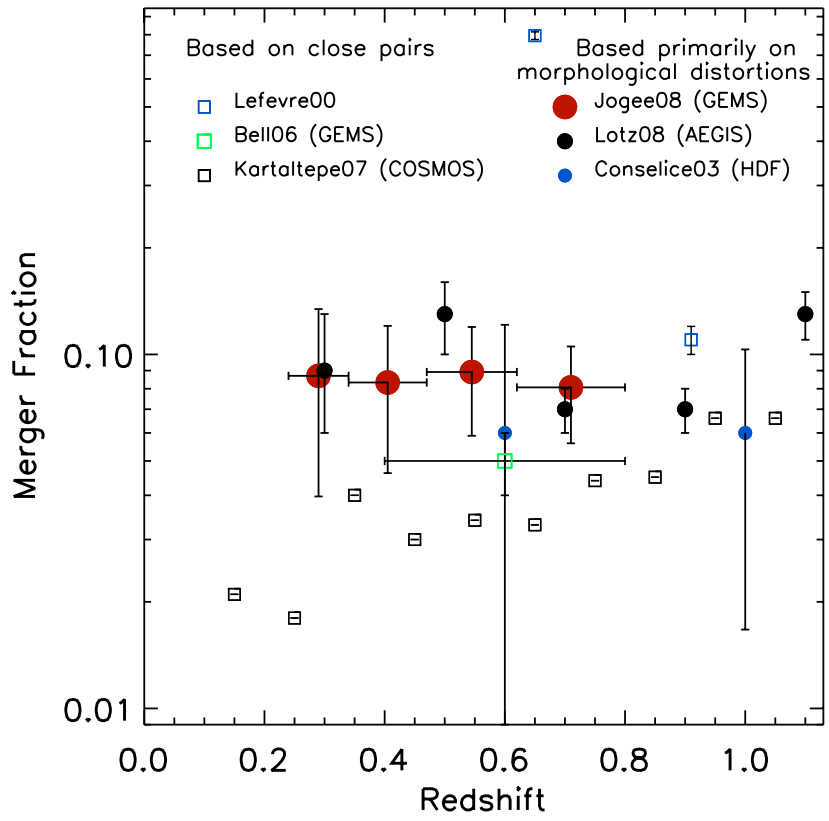

Figure 10. Observed merger fraction $f$ in the high-mass $\left(M \geqslant 2.5 \times 10^{10} M_{\odot}\right)$ sample is compared to other studies, noting the caveats outlined in Section 4.4. Shown here are the merger fraction based primarily on morphologically distorted galaxies (filled circles: this study; Lotz et al. 2008; Conselice 2003), and the close pair fraction (open squares: Le Fevre et al. 2000; Bell et al. 2006; Kartaltepe et al. 2007) as a function of redshift. See Section 4.4 for details.

(A color version of this figure is available in the online journal.)

are too large to set useful constraints at $z<1$ (Figure 10). Our results of a fairly flat evolution of the merger rate out to $z \sim 0.8$ also agree with the results of Cassata et al. (2005), which are based on both pairs and asymmetries.

Figure 10 also shows the result from three studies based on close pairs. The major merger fraction of massive galaxies $\left(M_{*} \geqslant 2.5 \times 10^{10} M_{\odot}\right)$ in close $(d<30 \mathrm{kpc})$ pairs, based on the 2-point correlation function in COMBO-17, is $5 \% \pm$ $1 \%$ averaged over at $0.4<z<0.8$ (Bell et al. 2006). This value is lower than our merger fraction $f(\sim 8 \% \pm 2 \%)$, which represent likely mergers of mass ratio $>1 / 10$, and it is higher than the fraction of cases we see as clear major mergers $(\sim 1.3 \% \pm 0.2 \%)$. The study of luminous $\left(L_{\mathrm{V}}>0.4 L_{*}\right)$ pairs at projected separations of 5-20 kpc in the COSMOS field (Kartaltepe et al. 2007) finds a galaxy pair fraction of $\sim 1 \%-3 \%$ over $z \sim 0.24-0.80$, corresponding to a galaxy merger fraction of $\sim 2 \%-6 \%$. Our observed fraction $f$ in the range of $9 \% \pm 5$ to $8 \% \pm 2 \%$ over $z \sim 0.24-0.8$ is slightly higher and flatter than this study.

The differences we see through these comparisons are already known (see Section 1). Studies based on close pairs tend to show moderate to fairly strong evolution in the major merger rate out to $z \sim 1.2$ (e.g., Kartaltepe et al. 2007; Bell et al. 2006; Lin et al. 2004), while studies based on asymmetries (e.g., Lotz et al. 2008; this study), and studies based on both pairs and asymmetries (Cassata et al. 2005) tend to report only mild evolution of the merger rate with redshift up to $z \sim 1$.

It is not fully understood why these different methods yield different results, but several factors likely play a part. First, it should be noted that the claim of strong evolution in the close pair fraction out to $z \sim 1.2$ in the COSMOS study by Kartaltepe et al. (2007) comes about when the low redshift $z \sim 0$ point from the SDSS pair catalog (Allam et al. 2004) is included in their analysis. The evolution within the internally consistent data set from COSMOS over $z \sim 0.15-1.05$ shows much weaker evolution (Figure 10). The drop in close pair fraction seems primarily to occur at $z<0.2$, but it is unclear how reliable the low $z \sim 0$ points are due to the small volume sampled and systematic effects between studies. A further reason for the difference could be due to the fact that the methods used in these studies trace different phases of an interaction, with the pair method tracing the potential pre-merger phase, while the method based on the distorted galaxies trace the later phases, including the merger and post-merger phases.

Another point is that both pair and asymmetry methods are imperfect ways of tracing the merger fraction. Methods tracing morphologically disturbed galaxies may capture some fly-by tidal interactions rather than mergers, and this effect would cause the fraction of interacting galaxies to overestimate the merger fraction. However, this effect is not a dominant one due to the following reason: interaction signatures typically persist for a visibility timescale of $0.5 \mathrm{Gyr}\left(T_{\mathrm{vis}}\right)$, and a flyby companion causing the distortion would still be within $100 \mathrm{kpc}$ of the disturbed galaxy, assuming an escape speed of $200 \mathrm{~km} \mathrm{~s}^{-1}$. The distorted galaxies we identify do not typically have such a fly-by companion, of mass ratio $>1 / 10$ and similar spectrophotometric redshift. In studies based on close pairs, one source of uncertainty is that even pairs with members at the same redshift may not become gravitationally bound in the future. This effect might cause pairs to overestimate the true major merger fraction. On the other hand, erroneous spectrophotometric redshifts can cause us to either overestimate or underestimate the true close pair fraction, with the latter effect being more likely. Corrections for this effect are uncertain and depend on the shape of the spectrophotometric redshift errors (e.g., see Bell et al. 2006 for discussion).

\subsection{Comparison of Galaxy Merger History with $\Lambda C D M$ Models}

We compare our empirical merger fraction $f$ (Figure 11) and merger rate $R$ (Figure 12) to predictions from different theoretical models of galaxy evolution in the context of a $\Lambda \mathrm{CDM}$ cosmology, including the halo occupation distribution (HOD) models of Hopkins et al. (2008); SAMs of Somerville et al. (2008), Bower et al. (2006), and Khochfar \& Silk (2006); and the cosmological smoothed particle hydrodynamics (SPH) simulations from Maller et al. (2006). The models were provided to us directly by the authors or co-authors of these individual studies.

We first briefly describe the general problem of calculating galaxy merger rates. Predicting the rate of mergers per comoving volume and per unit time between isolated dark matter (DM) halos within a $\Lambda$ CDM model is relatively straightforward via semi-analytic methods or $N$-body simulations (e.g., Lacey \& Cole 1993; Gottlöber et al. 2001; Fakhouri \& Ma 2008; Neistein \& Dekel 2008; D’Onghia et al. 2008). However, making a direct prediction of the galaxy merger rates is more complicated due to a number of factors, including the difference between the galaxy and halo merger timescales, tidal heating and stripping of halos and sub-halos, the effect of a dense core of baryons on merging satellites, and the nonlinear relation at low mass between DM halo (or sub-halo) mass and galaxy mass (van den Bosch et al. 2007). Thus, attempts to extract a galaxy merger rate from $\Lambda \mathrm{CDM}$ simulations also must attempt to model the relationship between dark matter and galaxy properties. The three main methods for making this connection are HOD models, SAMs, and hydrodynamic simulations. We summarize below how these three types of models differ. 


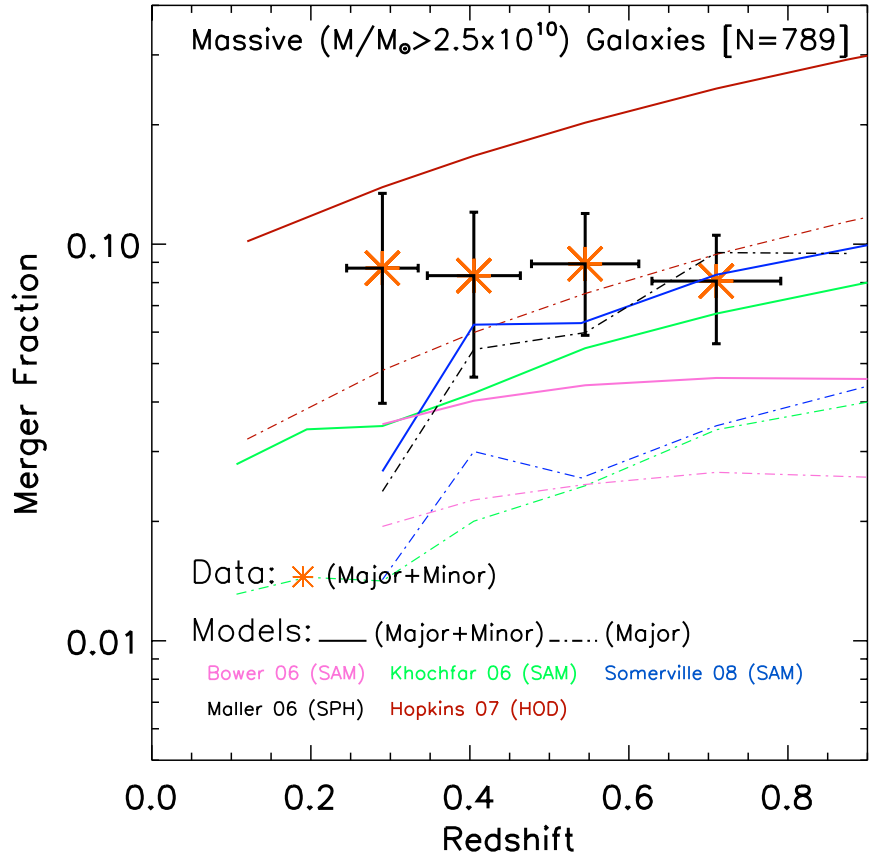

Figure 11. Empirical merger fraction $f$ (orange stars) for mergers with mass ratio $M 1 / M 2>1 / 10$ among high-mass galaxies is compared to the fraction of (major+minor) mergers (solid lines; stellar mass ratio $M 1 / M 2>1 / 10$ ) predicted by different $\Lambda$ CDM-based simulations of galaxy evolution, including the halo occupation distribution (HOD) models of Hopkins et al. (2008); SAMs of Somerville et al. (2008), Bower et al. (2006), and Khochfar \& Silk (2006); and smoothed particle hydrodynamics (SPH) cosmological simulations from Maller et al. (2006) (see Section 4.5).

(A color version of this figure is available in the online journal.)

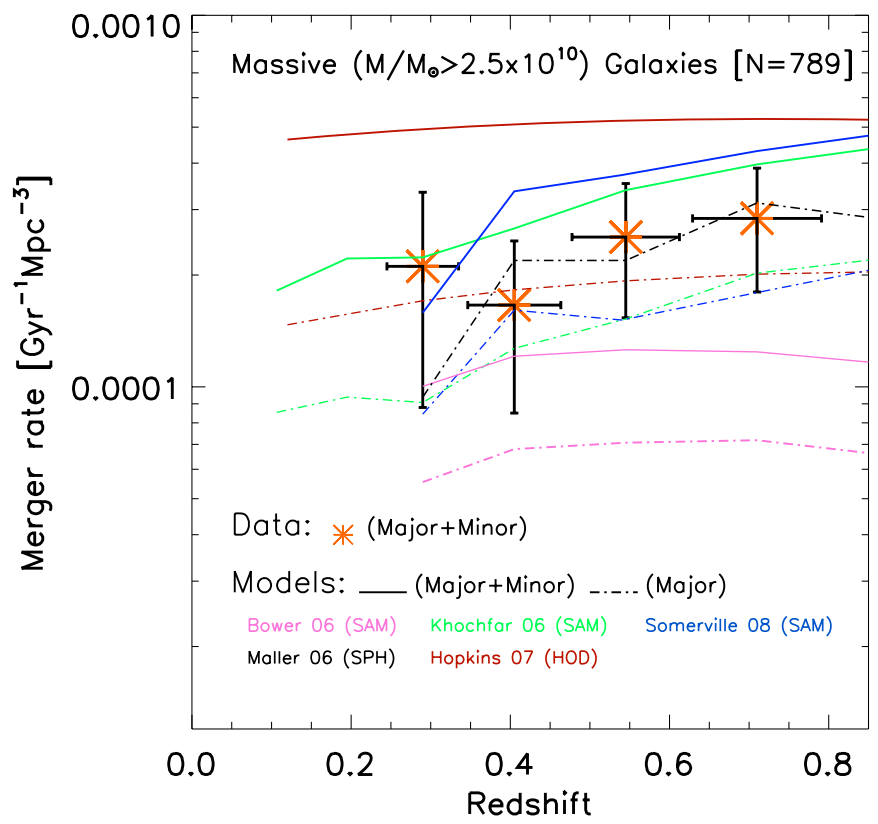

Figure 12. As in Figure 11, but now comparing the empirical rate $R$ (orange stars) of mergers with mass ratio $M 1 / M 2>1 / 10$ among high-mass galaxies to the rate of (major+minor) mergers (solid lines; stellar mass ratio $M 1 / M 2>1 / 10$ ) predicted by different $\Lambda \mathrm{CDM}$-based simulations of galaxy evolution.

(A color version of this figure is available in the online journal.)

HOD models specify the probability that a DM halo of a given mass $M$ harbors $N$ galaxies above a given mass or luminosity. The parameters of this function are determined by requiring that statistical observed quantities, such as galaxy mass or luminosity functions and galaxy correlation functions, be reproduced. The merger rate of galaxies within their host halos is calculated via standard or improved dynamical friction formulae. In the HOD models of Hopkins et al. (2008) used here, different modified formulae can be used, which include the effect of a gravitational capture cross section, stripping of DM halos, and calibration factors from $N$-body simulations. The predicted model rate can vary by a factor of $\sim 2$ depending on model assumptions for subhalo structure and mass functions, the halo occupation statistics, and the dynamical friction formulae used.

In SAMS, merger trees of DM halos are either extracted from cosmological $N$-body simulations or derived using analytic methods (e.g., Somerville \& Kolatt 1999). Calibrated modified versions of the Chandrasekhar dynamical friction approximation (e.g., Boylan-Kolchin et al. 2008) are used to compute the galaxy merger rate. Simplified analytic formulae are used to model the cooling of gas, SF, supernova feedback, and more recently, AGN feedback (e.g., Somerville et al. 2008; Bower et al. 2006; Croton et al. 2006; Benson et al. 2005; Cole et al. 2000; Somerville \& Primack 1999). The free parameters in these formulae are normalized to reproduce observations of nearby galaxies, such as the $z=0$ galaxy mass or luminosity function. Figure 12 shows results of three independent SAMs from Khochfar \& Silk (2006), Bower et al. (2006), and Somerville et al. (2008).

Cosmological hydrodynamic simulations attempt to model the detailed physics of gas hydrodynamics and cooling as well as gravity by explicitly solving the relevant equations for particles or grid cells. SPH methods are most commonly used. SF and supernova feedback are treated using empirical recipes. A drawback of this approach is that, due to computational limitations, state-of-the-art simulations still do not have the dynamic range to resolve the internal structure of galaxies while simultaneously treating representative cosmological volumes. Another well known problem is that cosmological SPH models, which do not include some kind of suppression of cooling (e.g., due to AGN feedback) in massive halos do not reproduce the observed number density of galaxies on the mass scales of interest (few $\times 10^{10} M_{\odot}$ ). Thus, the simulations of Maller et al. (2006) shown here, over-predict the number of high and low mass galaxies, while galaxies at the bend of the Schechter mass function are a factor of 2-3 too massive. In order to make the simulated mass function agree better with observations, Maller et al. (2006) apply a correction factor of 2.75 for galaxies in the mass range $2 \times 10^{10}<M_{*} / M_{\odot}<6 \times 10^{11}$. This correction is already included in the model on Figure 12.

When comparing the observations to the models, one must consider carefully how merger rates and fractions are determined in these simulations. Two approaches are used: one based on simulation snapshots and the other based on a light cone. In the first approach, simulation outputs ("snapshots") are stored at a sequence of redshifts. Two snapshots separated by a time $\Delta t$ are considered and modelers trace the merger history of galaxies whose final stellar mass $M_{*}$ is greater or equal to a given mass cut $M_{\text {cut }}$. The same mass cut $\left(M_{*} \geqslant 2.5 \times 10^{10}\right)$ used in the data is applied to the simulations. In order to mimic the observations as closely as possible, the interval $\Delta t$ in the model should ideally be equal to the visibility timescale $t_{\mathrm{vis}} .{ }^{19}$ One then counts the number $N_{1}$ of model galaxies with $M_{*} \geqslant M_{\text {cut }}$, which have experienced a merger of mass ratio $M 1 / M 2>1 / 10$ within the last $t_{\mathrm{vis}}$. It is important to note two points. Firstly, if a galaxy

\footnotetext{
${ }^{19}$ If $\Delta t$ is larger than $t_{\mathrm{vis}}$, a correction factor of order $\left(t_{\mathrm{vis}} / \Delta t\right)$ needs to be
} applied to the model merger fraction $f_{\bmod 1}$. 
were to undergo multiple mergers within a time $t_{\text {vis }}$, these mergers would be counted only once in the model term $N_{1}$, analogous to the case of the data where one cannot discriminate between multiple mergers within the time $t_{\mathrm{vis}}$ over which a galaxy appears distorted due to a merger. Secondly, the fact that $t_{\mathrm{vis}}$ is so short makes it very unlikely for a model galaxy to undergo more than one merger over this timescale. As a result the model term $N_{1}$ is essentially tracing the number $N_{\mathrm{mrg} 1}$ of mergers within the last $t_{\mathrm{vis}}$. One can then determine the merger rate using $R_{\bmod 1}=$ $N_{\operatorname{mrg} 1} /(\Delta t V)$, where $V$ is the comoving volume of the simulation box. The merger fraction is $f_{\bmod 1}=N_{\mathrm{mrg} 1} / N_{\text {gal } 1}$, where $N_{\text {gal }}$ is the total number of galaxies above the relevant mass limit. Except for Somerville et al. (2008), all the models presented on Figure 11 and Figure 12 derive the merger fraction $f$ and rate $R$ using the above approach, based on simulation snapshots.

For the Somerville et al. (2008) models, the simulation analysis was carried out in a way that is closer to the observations. We construct a light cone with a geometry that is equivalent to three GEMS fields ( $2700 \operatorname{arcmin}^{2}$ from $0.1<z<1.1$ ). We then divide the galaxies into redshift bins, exactly as in the observational analysis, and we count the number $N_{\mathrm{mrg} 2}$ of galaxies that have had a merger within a time $t_{\mathrm{vis}}$ in the past. These galaxies appear as morphologically distorted mergers in the observations. The model merger fraction is $f_{\bmod 2}=N_{\mathrm{mrg} 2} / N_{\mathrm{gal} 2}$, where $N_{\text {gal2 }}$ is the total number of galaxies above the relevant mass limit in this light cone or redshfit bin. The model merger rate is calculated exactly as in the data using $R_{\bmod 2}=f_{\bmod 2} n_{\bmod 2} / t_{\mathrm{vis}}$, where $n_{\bmod 2}$ is the comoving number density of galaxies above a certain mass limit in the redshift bin. Note that $N_{\operatorname{mrg} 2}$ and $f_{\bmod 2}$ are quite sensitive to $t_{\mathrm{vis}}$, while $R$ is independent of $t_{\mathrm{vis}}$.

In Figure 11, we compare the empirical merger fraction $f$ to the corresponding model predictions $f_{\bmod 1}$ and $f_{\bmod 2}$. The comparison between the empirical merger rate $R$ (Equation (3) in Section 4.3) and the model predictions $R_{\bmod 1}$ and $R_{\bmod 2}$ is in Figure 12. In both data and models, major and minor mergers are defined as those with mass ratio $(M 1 / M 2>1 / 4)$, and (1/ $10 \leqslant M 1 / M 2<1 / 4)$, respectively. The only slight exception is in the case of Maller et al. (2006) model where the extracted major mergers were defined with a slightly lower mass cutoff $(M 1 / M 2>1 / 3)$. The dotted lines on Figures 11 and 12 show the major merger rate for all the models. The solid line show the (major+minor) merger rate, in other words, the rate of mergers with mass ratio $(M 1 / M 2>1 / 10)$. This is shown for all models except the Maller et al. (2006) SPH simulations, where the limited dynamic range of the current simulations only allows predictions for major mergers. The solid line can be directly compared to our empirical merger fraction $f$ or rate $R$, since the latter refer to mergers of mass ratio $M 1 / M 2>1 / 10$.

We find qualitative agreement between the observations and models, such that the (major+minor) merger fraction (Figure 11) and merger rate (Figure 12) from different models (solid lines) bracket the corresponding empirical estimates (stars) and show a factor of 5 dispersion. One can now anticipate that in the near future, improvements in both the observational estimates and model predictions will start to rule out certain merger scenarios and refine our understanding of the merger history of galaxies.

\subsection{The Impact of Galaxy Mergers on the Average SFR Over the Last 7 Gyr}

Both observations (e.g., Larson \& Tinsley 1978; Joseph \& Wright 1985; Kennicutt et al. 1987; Barton Gillespie et al. 2003) and simulations (e.g., Negroponte \& White 1983; Hernquist 1989; Barnes \& Hernquist 1991, 1996; Mihos \& Hernquist 1994, 1996; Springel et al. 2005b) suggest that galaxy interactions and mergers trigger SF. However, simulations cannot uniquely predict the factor by which mergers enhance the SF activity of galaxies over the last $7 \mathrm{Gyr}$, since the SFR in simulations is highly sensitive to the stellar feedback model, the bulge-todisk $(B / D)$ ratio, the gas mass fractions, and orbital geometry (e.g., Cox et al. 2006; di Matteo et al. 2007). Thus, we explore here the impact of interactions on the average UV-based and $\mathrm{UV}+\mathrm{IR}$-based SFR of intermediate-to-high-mass $\left(M \geqslant 1 \times 10^{9}\right.$ $M_{\odot}$ ) galaxies over $z \sim 0.24-0.80$.

We adopt the SFRs in Bell et al. (2005, 2007), based on COMBO-17 UV data (Wolf et al. 2004) and deep Spitzer $24 \mu \mathrm{m}$ observations with a limiting flux of $\sim 83 \mu \mathrm{Jy}(5 \sigma)$ from the Spitzer Guaranteed Time Observers (Papovich et al. 2004; Gordon et al. 2005). The unobscured SFR based on the directly observable UV light from young stars was computed using $\mathrm{SFR}_{\mathrm{UV}}=9.8 \times 10^{-11}\left(2.2 L_{\mathrm{UV}}\right)$, where $L_{\mathrm{UV}}=1.5 v l_{v, 2800}$ is a rough estimate of the total integrated 1216-3000 $\AA \mathrm{UV}$ luminosity, derived using the $2800 \AA$ rest-frame luminosity from COMBO-17 $l_{v, 2800}$. The factor of 1.5 used in converting the $2800 \AA$ luminosity to total UV luminosity accounts for the UV spectral shape of a 100 Myr old population with constant SFR. The factor of 2.2 corrects for the light emitted longward of 3000 $\AA$ and shortward of $1216 \AA$. The SFR calibration is derived from Pégase assuming a $100 \mathrm{Myr}$ old stellar population with constant SFR and a Chabrier (2003) IMF.

The obscured SFR can be calculated from dust-reprocessed IR emission using the expression $\mathrm{SFR}_{\mathrm{IR}}=9.8 \times 10^{-11} L_{\mathrm{IR}}$, where $L_{\mathrm{IR}}$ is the total IR luminosity (TIR) over 8-1000 $\mu \mathrm{m}$ (Bell et al. 2007). $L_{\mathrm{IR}}$ is constructed from the observed $24 \mu \mathrm{m}$ flux (corresponding to rest-frame wavelengths of 19-13 $\mu \mathrm{m}$ over $z \sim 0.24-0.80$ ) using the method outlined in Papovich \& Bell (2002), based on an average Sbc template from the Devriendt et al. (1999) SED library. In converting from $L_{\mathrm{IR}}$ to $S F R_{\mathrm{IR}}$, Bell et al. (2007) assume that the bulk of the $24 \mu \mathrm{m}$ emission comes from SF, and not from AGN activity, based on the statistical result that less than $15 \%$ of the total $24 \mu \mathrm{m}$ emission at $z<1$ is in X-ray luminous AGNs (e.g., Silva et al. 2004; Bell et al. 2005; Franceschini et al. 2005; Brand et al. 2006). Uncertainties in these SFR estimates are no less than a factor of 2 for individual galaxies while the systematic uncertainty in the overall SFR scale is likely to be less than a factor of 2 (Bell et al. 2007).

We investigate the SF properties of the sample S1 of $\sim 789$ high-mass $\left(M \geqslant 2.5 \times 10^{10} M_{\odot}\right)$ and the sample $\mathrm{S} 2$ of $\sim 3698$ intermediate-mass $\left(M \geqslant 1.0 \times 10^{9} M_{\odot}\right)$ galaxies. As described in Section 2, the high-mass sample $\mathrm{S} 1$ is complete for both the red sequence and blue cloud, while the intermediate-mass sample S2 is only complete for the blue cloud and suffers from incompleteness on the red sequence in the highest redshift bins. However, since most of the SFR density originates from the blue cloud, this incompleteness does not have any major impact on the results. Figure 13 shows the UV-based SFR plotted versus the stellar mass in each redshift bin. The UV-based SFR ranges from $\sim 0.01$ to $25 M_{\odot} \mathrm{yr}^{-1}$, with most galaxies having a rate below $5 M_{\odot} \mathrm{yr}^{-1}$.

While it is desirable to use the Spitzer $24 \mu \mathrm{m}$ data in order to account for obscured SF, only $\sim 24 \%$ ( $\sim 878$ galaxies) of the 3698 galaxies in our intermediate-mass sample have a Spitzer $24 \mu \mathrm{m}$ detection, although over $86 \%$ of the sample is covered by the Spitzer observations down to a limiting flux of $\sim 83 \mu \mathrm{Jy}$. The detected galaxies yield a median ratio of $\left(\mathrm{SFR}_{\mathrm{IR}} / \mathrm{SFR} \mathrm{UV}\right)$ 


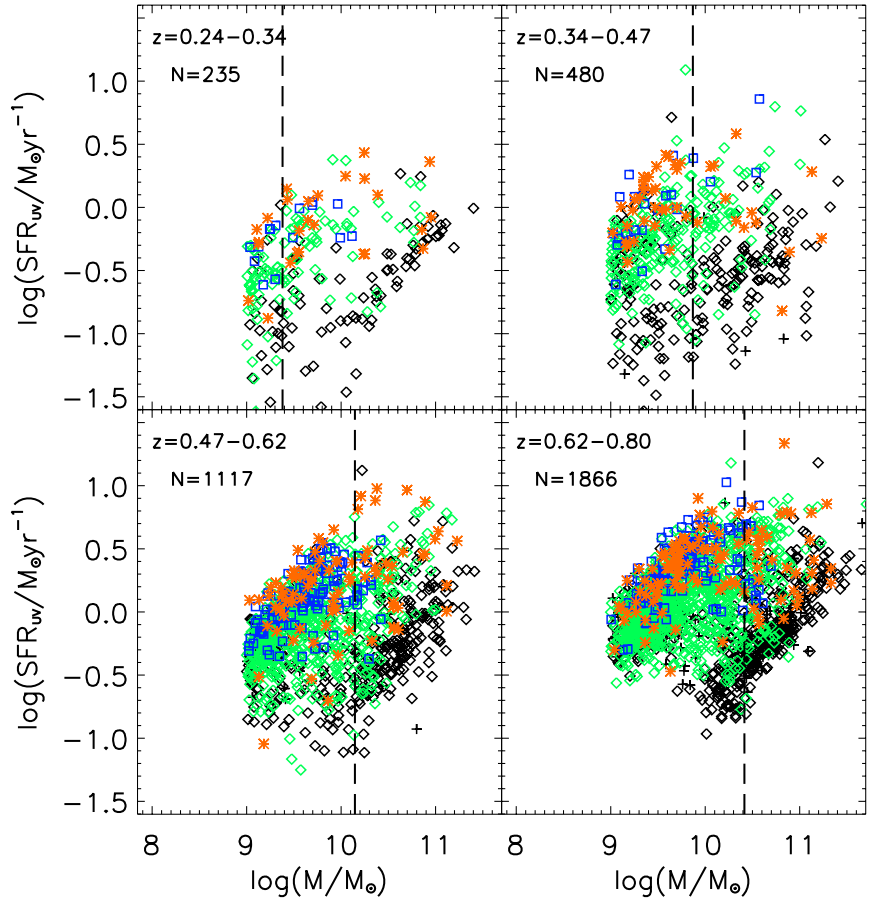

Figure 13. UV-based SFR is plotted vs. the stellar mass over $z \sim 0.24-0.80$ for the sample S2 of galaxies with $M \geqslant 1 \times 10^{9} M_{\odot}$. The four panels show the four redshift bins, which span $1 \mathrm{Gyr}$ each, and cumulatively cover the interval $z \sim 0.24-0.80\left(T_{\text {back }} \sim 3-7 \mathrm{Gyr}\right) . N$ denotes the number of galaxies plotted in each bin. Galaxies are coded as in Figure 1, with merging systems denoted by orange stars. The average SFR and total SFR density in both the UV and the IR, are further illustrated in Figures 14 and 16.

(A color version of this figure is available in the online journal.)

of $\sim 3.6$, indicative of a sustantial amount of obscured SF. Three of the interacting galaxies in the first redshift bin had anomalously high $\operatorname{SFR}_{\mathrm{UV}+\mathrm{IR}}\left(\sim 41,18\right.$, and $\left.15 M_{\odot} \mathrm{yr}^{-1}\right)$. Two of these turned to have infrared spectra consistent with an AGN and were removed before computing the IR-based SF properties shown in Figures 14-21.

The average UV-based SFR (based on 3698 galaxies) and UV+IR-based SFR (based on only the 876 galaxies with 24 $\mu \mathrm{m}$ detections) are plotted in the top 2 panels of Figure 14 for three groups of intermediate-mass galaxies: mergers, noninteracting E-Sd, and non-interacting Irr1. The corresponding plot for the high-mass sample is in Figure 15. It can be seen (Figures 14 and 15) that over $z \sim 0.24-0.80$, the average UVbased and UV+IR-based SFR of mergers (in the phase where they are recognizable as mergers) are only modestly enhanced, at best by a factor of a few, compared to the non-interacting galaxies. This result applies to both high-mass $(M \geqslant 2.5 \times$ $\left.10^{10} M_{\odot}\right)$ galaxies and intermediate-mass $\left(M \geqslant 1.0 \times 10^{9} M_{\odot}\right)$ blue-cloud galaxies. A similar result is also found by Robaina et al. (2009, in preparation) in high-mass systems. This modest enhancement is consistent with the recent statistical study of di Matteo et al. (2007), who find from numerical simulations of several hundred galaxy collisions that the maximum SFR in galaxy mergers is typically only a factor of 2-3 larger than that of corresponding non-interacting galaxies. Their results suggest that the results of some early simulations (e.g., Mihos \& Hernquist 1996; Hernquist \& Mihos 1995), where mergers converted 50-80 percent of their original gas mass into stars, may not represent the typical situation at $z<1$.

In order to further test the robustness of our result, we used the stacking procedure described in Zheng et al. (2006) to get

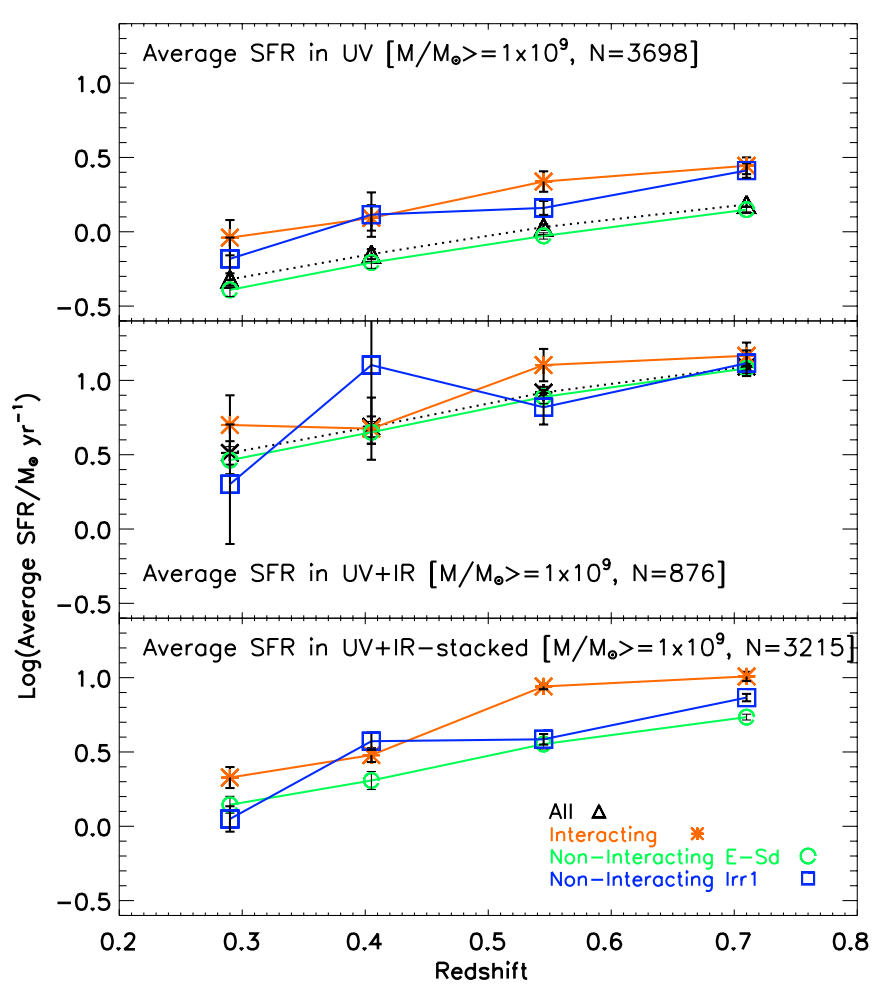

Figure 14. For the sample $\mathrm{S} 2$ of galaxies with $M \geqslant 1 \times 10^{9} M_{\odot}$, the average SFR of merging systems, non-interacting E-Sd galaxies, and non-interacting Irr 1 galaxies are compared over $z \sim 0.24-0.80$. $N$ denotes the number of galaxies used. The average UV-based SFR (top panel; based on 3698 galaxies), average $\mathrm{UV}+\mathrm{IR}$-based SFR (middle panel; based on only the 876 galaxies with $24 \mu \mathrm{m}$ detections), and average UV+IR-stacked SFR (based on 3215 galaxies with 24 $\mu \mathrm{m}$ coverage) are shown. In all there cases, the average SFR of visibly merging systems is only modestly enhanced compared to non-interacting galaxies over $z \sim 0.24-0.80$ (lookback time 3-7 Gyr). See Section 4.6 for details.

(A color version of this figure is available in the online journal.)

a more representative measure of the IR-based SFR for the following three groups of intermediate-mass systems: mergers, non-interacting E-Sd, and non-interacting Irr1 galaxies. For every group, the individual galaxies were cross-correlated with the Spitzer $24 \mu \mathrm{m}$ catalog in order to identify detected and undetected objects. Then the PSF-removed $24 \mu \mathrm{m}$ images for the undetected objects were stacked, and a mean flux was derived from the average/median stacked image. An average $24 \mu \mathrm{m}$ luminosity was determined from the individually detected fluxes and individually undetected fluxes estimated by stacking. The 3215 intermediate-mass galaxies in the Spitzer field were used in this process, giving a more representative $24 \mu \mathrm{m}$ luminosity than the mere 878 galaxies with detections. A final uncertainty can be obtained by combining background error and bootstrap error in quadrature. The IR-based SFR was estimated from the $24 \mu \mathrm{m}$ luminosity using the procedure described above, and combined with the UV-based SFR to estimate the total SFR. The average UV+IR-stacked SFR is plotted in the bottom panel of Figures 14 and 15: again, only a modest enhancement is seen in the average SFR of mergers (in the phase where they are recognizable as mergers), compared to non-interacting galaxies.

\subsection{The Contribution of Interacting Galaxies to the Cosmic SFR Density Over the Last 7 Gyr}

Over the last 8 Gyr since $z \sim 1$, the cosmic SFR density is observed to decline by a factor of 4-10 (e.g., Lilly et al. 1996; Ellis et al. 1996; Flores et al. 1999; Haarsma et al. 2000; Hopkins 


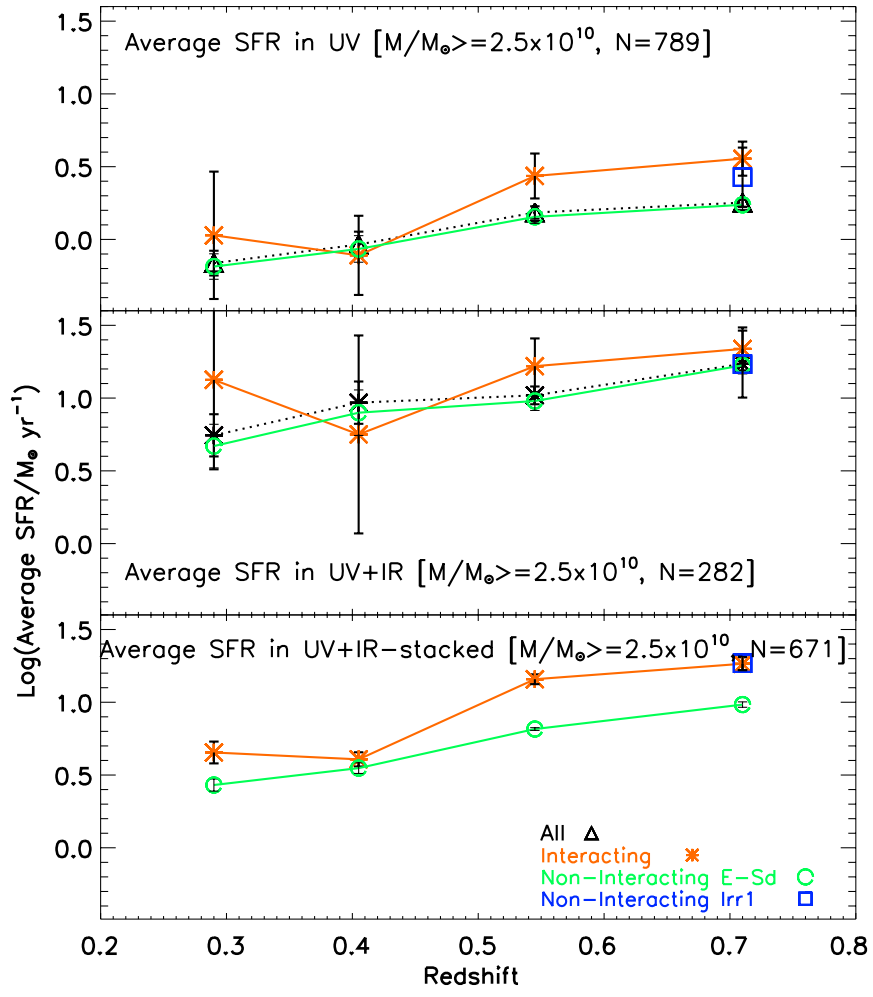

Figure 15. As in Figure 14, but for the sample S1 of high-mass $(M \geqslant 2.5 \times$ $10^{10} M_{\odot}$ ) galaxies. Only data points with at least three galaxies are shown. Again, the average SFR of visibly merging galaxies is only modestly enhanced compared to non-interacting galaxies over $z \sim 0.24-0.80$ (lookback time $\sim$ 3-7 Gyr).

(A color version of this figure is available in the online journal.)

2004; Pérez-González et al. 2005; Le Floc'h et al. 2005). Earlier GEMS studies by Wolf et al. (2005) and Bell et al. (2005) over a 0.6 Gyr interval $\left(z \sim 0.65-0.75\right.$ or $\left.T_{\mathrm{b}} \sim 6.2-6.8 \mathrm{Gyr}\right)$ showed that the UV and IR luminosity density over this interval are dominated by non-interacting galaxies. Here, we extend the earlier GEMS studies to cover a six-fold larger time interval of 4 Gyr $\left(z \sim 0.24-0.80\right.$ or $\left.T_{\mathrm{b}} \sim 3-7 \mathrm{Gyr}\right)$, and set quantitative limits on the contribution of merging systems to the UV-based and UV+IR-based SFR density. We use the sample S2 of $\sim 3698$ intermediate-mass $\left(M \geqslant 1.0 \times 10^{9} M_{\odot}\right)$ galaxies. Our study also complements the IR-based studies by Hammer et al. (2005; 195 galaxies at $z>0.4$ ) and Melbourne et al. (2005; 800 galaxies) in terms of sample size or/and SFR indicators.

Figure 16 shows the SFR density for intermediate-mass mergers, non-interacting E-Sd galaxies, and non-interacting Irr 1 galaxies over $z \sim 0.24-0.80$. The top panel shows the UVbased SFR density from the full sample. The middle panel show the UV+IR-based SFR density from the 878 galaxies with individual $24 \mu \mathrm{m}$ detections. Finally, the bottom panel shows the UV+IR-stacked SFR density determined via the stacking of 3215 galaxies with Spitzer coverage, as outlined in Section 4.6. In all three panels, one finds that interacting galaxies only account for a small fraction $(<30 \%)$ of the cosmic SFR density over $z \sim 0.24-0.80$, corresponding to lookback times of 3-7 Gyr (Figure 16). The same results hold for the sample of high-mass $\left(M \geqslant 2.5 \times 10^{10} M_{\odot}\right)$ galaxies, as illustrated in Figure 17.

Thus, our results suggest that the behavior of the cosmic SFR density over $z \sim 0.24-0.80$ is predominantly shaped by noninteracting galaxies. Similar results are reported by Lotz et al. (2008) and Sobral et al. (2009), with the latter study reporting

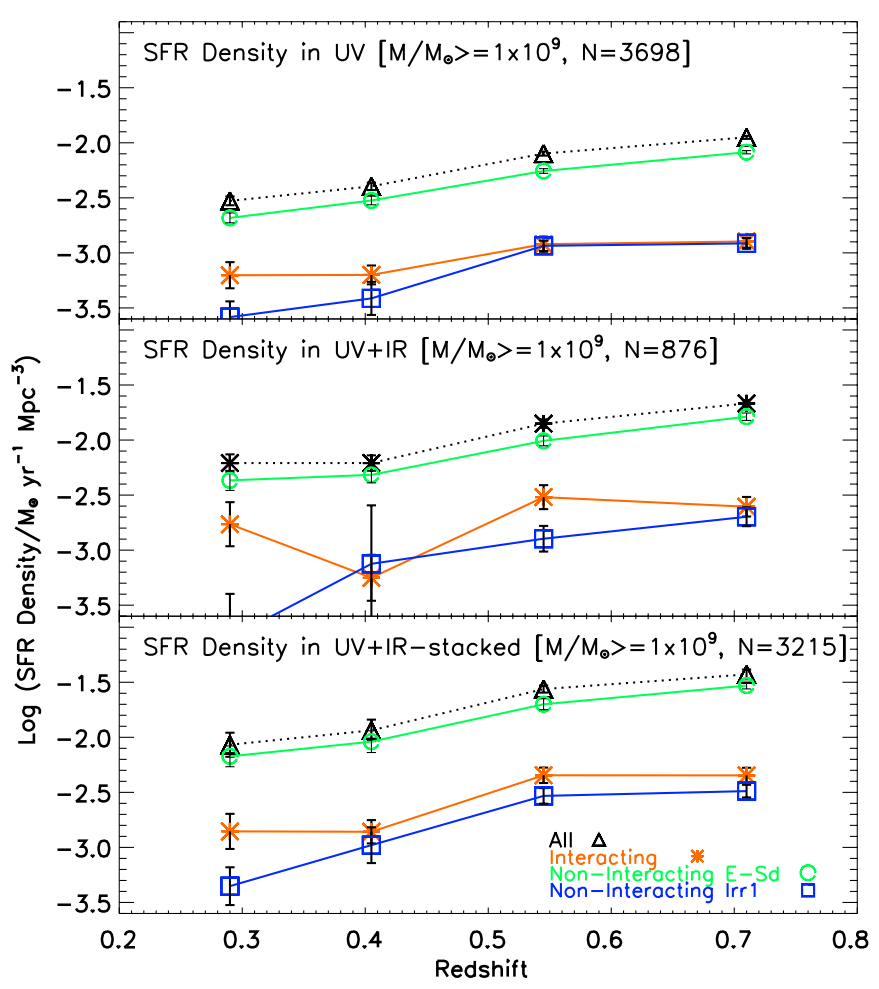

Figure 16. For the sample $\mathrm{S} 2$ of galaxies with $M \geqslant 1 \times 10^{9} M_{\odot}$, the SFR density of merging systems, non-interacting E-Sd galaxies, and non-interacting Irr galaxies are compared over $z \sim 0.24-0.80$. Results based on UV (top panel), UV+IR (middle panel), as well as UV+stacked-IR data (bottom panel), are shown in the top, middle, and bottom panels. In all bins, visibly merging systems only contribute a small fraction (typically below 25\%) of the total SFR density. In effect, the behavior of the cosmic SFR density over the last 7 Gyr is predominantly shaped by non-interacting E-Sd galaxies rather than visibly merging galaxies.

(A color version of this figure is available in the online journal.)

that $20 \%$ to $30 \%$ of the SFR density at $z \sim 0.84$ originates from mergers. Our result is a direct consequence of the fact that the merger fraction $f$ (Table 2; Section 4.3), as well as the enhancement in the average SFR from interactions (Section 4.6), are both modest. Our results agree remarkably well with models for the self-regulated growth of supermassive black holes in mergers involving gas-rich galaxies (Hopkins et al. 2006). These models predict that galaxy mergers contribute only $\sim 20 \%$ of the SFR density out to $z \sim 1$, and even out to $z \sim 2$.

It is legitimate to ask whether the results hold despite the uncertainties in identifying mergers. We first note that based on the tests of Section 4.1, we have already included a large fractional error term on $f$ to account for the binomial standard deviation, the dispersion between classifiers, and the effect of moderate bandpass shifting, and surface brightness dimming. Therefore, the results presented here already take into account at least some of these sources of uncertainties.

Another source of uncertainty might be that some of the galaxies, which we have classified as non-interacting Irr1 under the assumption that their small-scale asymmetries are likely caused by SF rather than interactions (Section 3.3), may be borderline cases of mergers or interacting systems. However, it can be seen from Figure 16, that even if we were to add the SFR density of all the non-interacting Irr1 galaxies to that of the mergers, the sum would still be significantly lower than the contribution of non-interacting E to Sd galaxies. Thus, the results would be largely unchanged. 


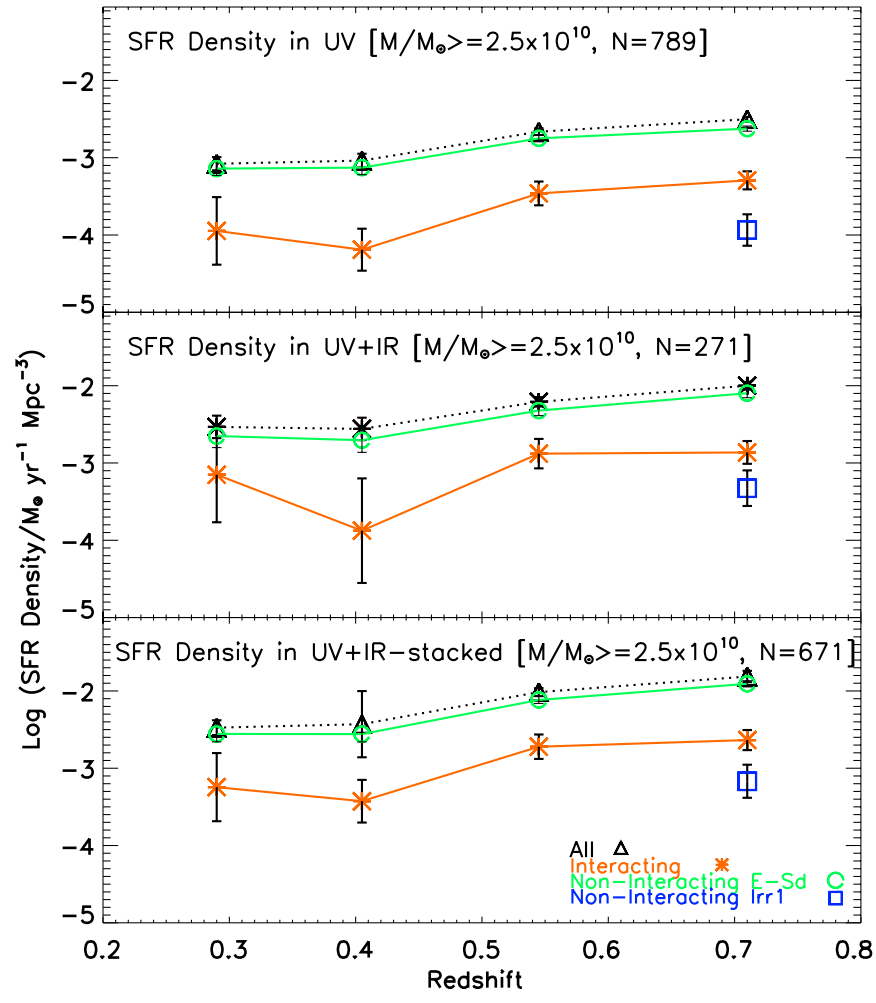

Figure 17. As in Figure 16, but for the sample of high-mass $(M \geqslant 2.5 \times$ $10^{10} M_{\odot}$ ) galaxies. Only data points with at least three galaxies are shown. The same conclusion holds: the cosmic SFR density over the last 7 Gyr is predominantly shaped by non-interacting E-Sd galaxies rather than visibly merging galaxies.

(A color version of this figure is available in the online journal.)

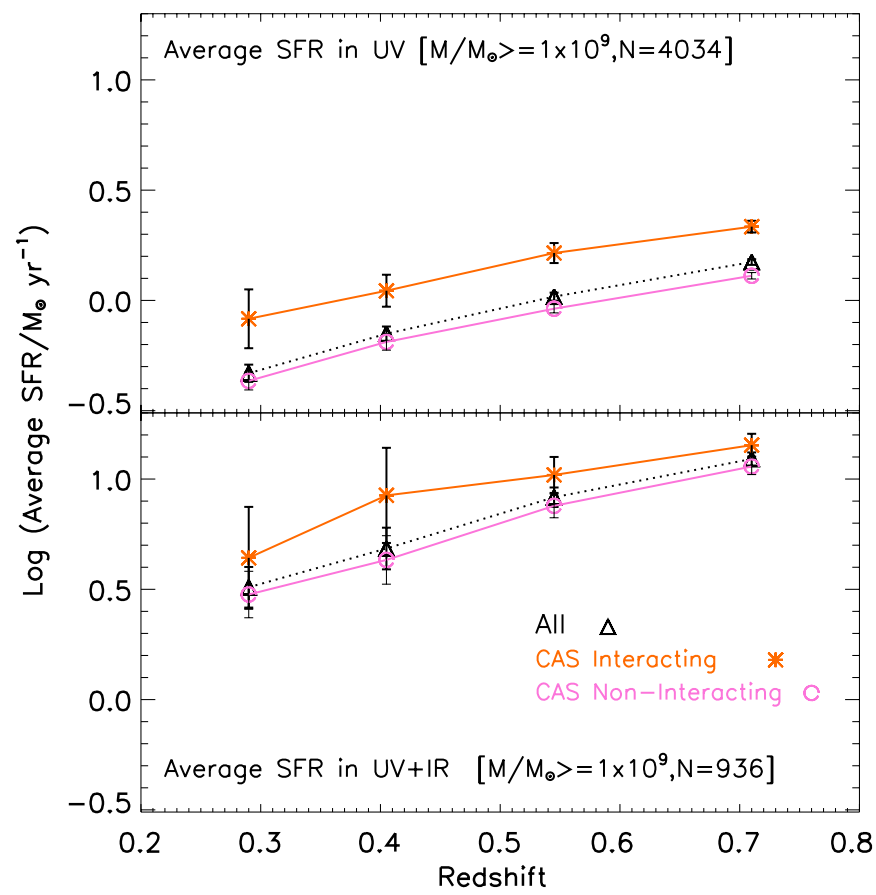

Figure 18. Same as in Figure 14, but using the CAS criterion $(A>0.35$ and $A>S)$ to identify interacting systems in the sample of intermediate-mass $(M \geqslant$ $1.0 \times 10^{9} M_{\odot}$ ) galaxies. The average SFR of "CAS-interacting" galaxies is only modestly enhanced compared to "CAS non-interacting" galaxies, in agreement with the results from Section 4.6.

(A color version of this figure is available in the online journal.)

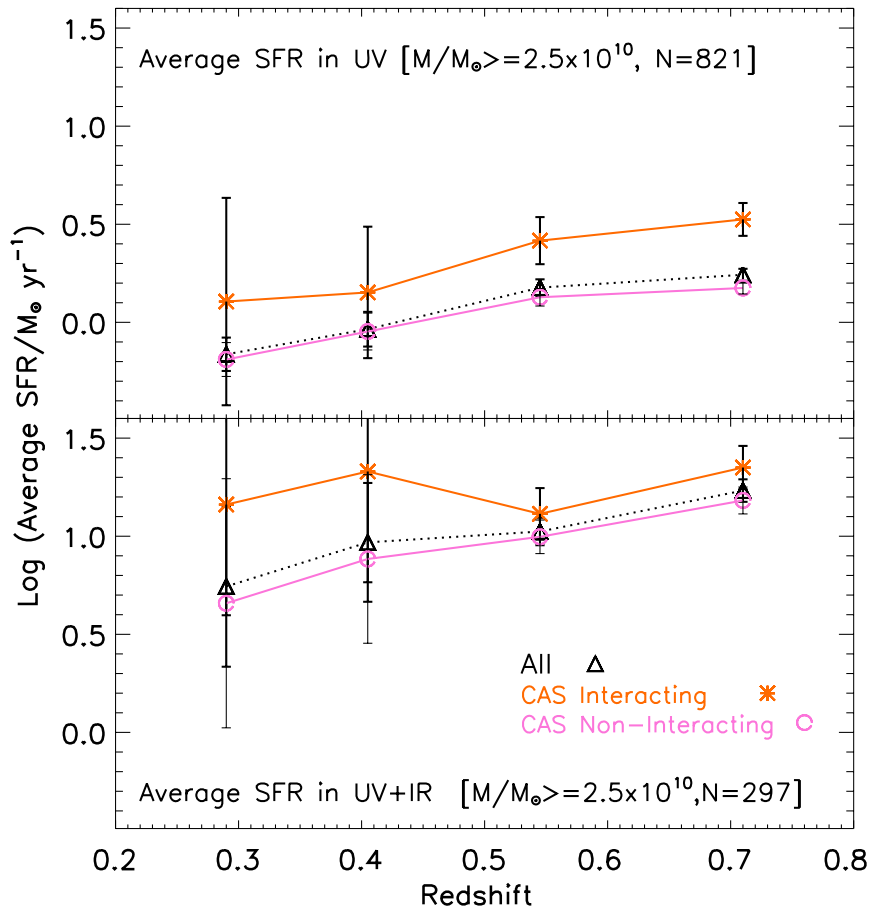

Figure 19. As in Figure 18, but for the sample of high-mass $\left(M \geqslant 2.5 \times 10^{10} M_{\odot}\right)$ galaxies. The same conclusion holds: the average SFR of "CAS-interacting" galaxies is only modestly enhanced compared to "CAS non-interacting" galaxies.

(A color version of this figure is available in the online journal.)

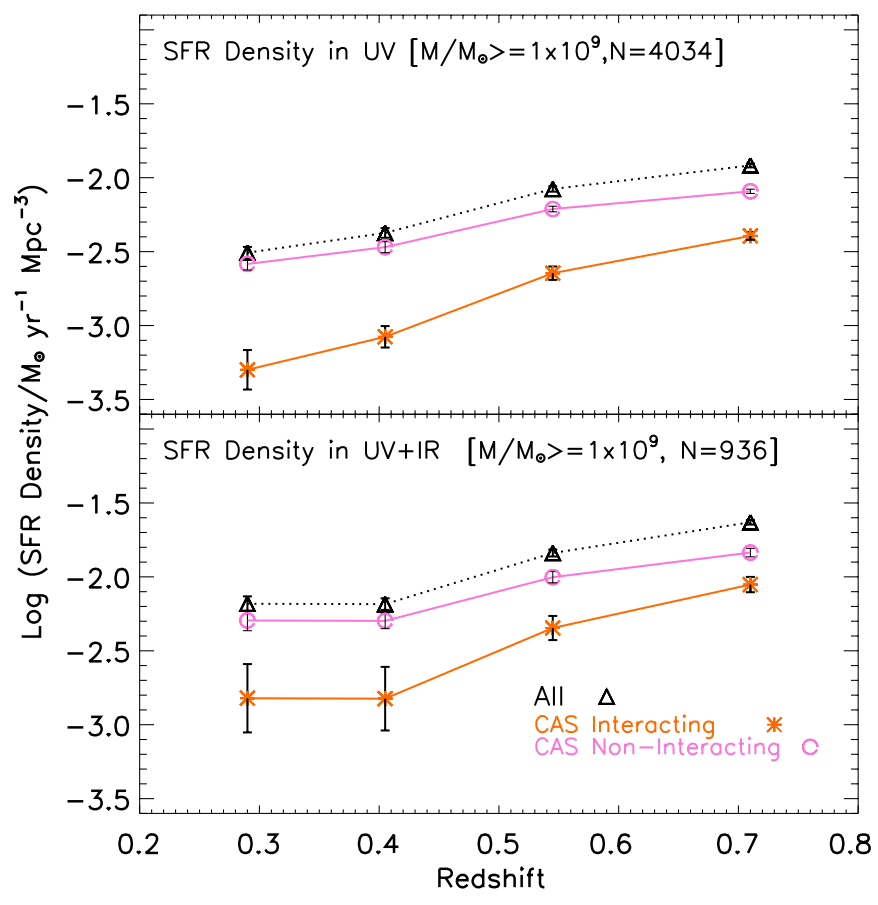

Figure 20. Same as in Figure 16, but using the CAS merger criterion $(A>0.35$ and $A>S$ ) to identify interacting galaxies in the sample of intermediate-mass $\left(M \geqslant 1.0 \times 10^{9} M_{\odot}\right)$ galaxies. The "CAS-interacting" galaxies contribute only $16 \%-33 \%$ of the UV SFR density and $22 \%-38 \%$ of the UV+IR SFR density. While the upper limits of these values are slightly higher than those based on the visual types (Figure 16), the "CAS non-interacting" galaxies' clearly dominate the SFR density.

(A color version of this figure is available in the online journal.) 


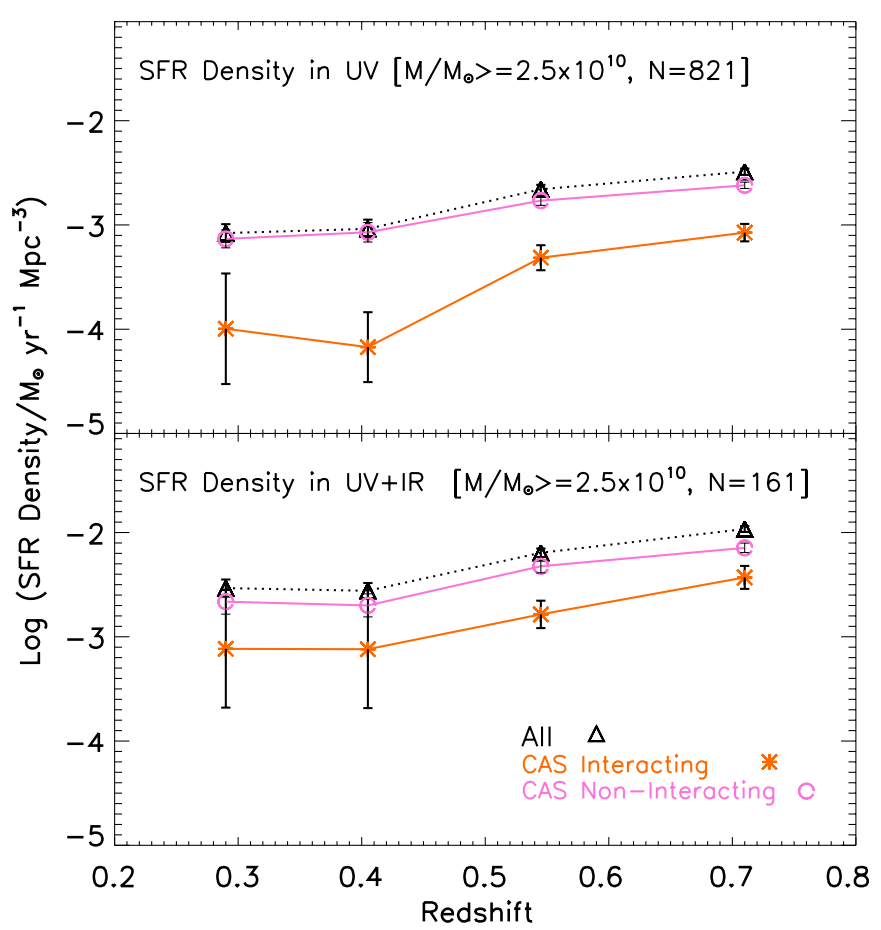

Figure 21. As in Figure 20, but for the sample of high-mass $\left(M \geqslant 2.5 \times 10^{10}\right.$ $\left.M_{\odot}\right)$ galaxies. The same conclusion holds.

(A color version of this figure is available in the online journal.)

Another test is to repeat the analyses using the CAS merger criterion $(A>0.35$ and $A>S)$ to identify mergers. The limited recovery rate $(50 \%-73 \%)$ and large level of contamination impacting the CAS criterion (Section 3.4) make it more difficult to interpret the SF properties of systems identified as mergers or non-interacting with CAS. For both the intermediate-mass sample (Figure 18) and the high-mass sample (Figure 19), the average SFR of CAS mergers is only modestly enhanced compared to CAS non-interacting galaxies, in agreement with the results from Section 4.6.

Furthermore, for the intermediate-mass sample, Figure 20 shows that CAS mergers contribute only $16 \%-33 \%$ of the UV SFR density and $22 \%-38 \%$ of the UV+IR SFR density. While the upper limits of these values are slightly higher than those based on the visual types, it is nonetheless reassuring that CAS non-interacting galaxies dominate the SFR density. Similar results hold for the sample of high-mass $\left(M \geqslant 2.5 \times 10^{10} M_{\odot}\right)$ galaxies, as illustrated in Figure 21.

For intermediate-mass $\left(M \geqslant 1.0 \times 10^{9} M_{\odot}\right)$ galaxies, we find that the cosmic SFR density declines by a factor of $\sim 3$ from $z \sim$ 0.80 to 0.24 (lookback time $\sim 7-3 \mathrm{Gyr}$ ). Since non-interacting galaxies dominate the cosmic SFR density in every redshift bin, it follows that this decline is largely the result of a shutdown in the SF of non-interacting galaxies. The question of what drives this shutdown will be addressed in detail in a future paper, and is only considered briefly here. One possibility is the depletion of the internal cold gas supply of galaxies by SF, or the reduction in the accretion rate of gas from cosmological filaments. Future facilities like ALMA will be instrumental in exploring this issue further. Another related possibility is that over time, most of the SFR is shifting to lower stellar masses. High-mass systems are associated with a lower SSFR (Cowie et al. 1996; Brinchmann et al. 2004; Brinchmann \& Ellis 2000; Fontana et al. 2003; Bauer et al. 2005; Zheng et al. 2007; Figure 1 of Noeske et al. 2007a), consistent with the idea that they have experienced the bulk of their stellar mass growth at earlier epochs $(z>1)$. In staged SF models (Noeske et al. 2007b) the SF history of low mass systems is consistent with exponential SF models associated with a late onset and a long duration.

\section{SUMMARY AND CONCLUSIONS}

We have performed a comprehensive observational estimate of the galaxy merger fraction over $z \sim 0.24-0.80$ (lookback times of 3-7 Gyr), and explored the impact of mergers on the SF of galaxies over this interval. Our study is based on HST ACS, COMBO-17, and Spitzer $24 \mu \mathrm{m}$ data from the GEMS survey. We use a large sample of $\sim 3600\left(M \geqslant 1 \times 10^{9} M_{\odot}\right)$ galaxies and $\sim 790$ high-mass $\left(M \geqslant 2.5 \times 10^{10} M_{\odot}\right)$ galaxies (Section 2$)$. We primarily identify mergers using a visual classification system, which is based on visual morphologies, spectrophotometric redshifts, and stellar masses (Sections 3.2 to 3.3), and identifies systems that show evidence of having experienced a merger of mass ratio $M 1 / M 2>1 / 10$ within the last visibility timescale. While many earlier studies focused only on major mergers (defined as mergers with $M 1 / M 2>1 / 4$ ), we also attempt to constrain the frequency of minor mergers (defined as mergers with $1 / 10<M 1 / M 2 \leqslant 1 / 4)$, since they dominate the merger rates in $\Lambda \mathrm{CDM}$ models. Below is a summary of our results:

1. For the high-mass $\left(M \geqslant 2.5 \times 10^{10} M_{\odot}\right)$ sample of $\sim 790$ galaxies, which is complete on both the blue cloud and red sequence, we find the following. The fraction $f$ of visually classified systems that show evidence of a recent merger of mass ratio $>1 / 10$, does not show strong evolution over lookback times of 3-7 Gyr, and ranges from $9 \% \pm$ $5 \%$ at $z \sim 0.24-0.34$, to $8 \% \pm 2 \%$ at $z \sim 0.60-0.80$ (Table 1; Figure 4). These mergers are further subdivided into three categories: clear "major merger", clear "minor merger", and ambiguous "major or minor merger" cases. The first two classes are used to set lower limits on the major and minor merger fraction. The lower limit on the major merger fraction, determined in this way, ranges from $1.1 \%$ to $3.5 \%$ over $z \sim 0.24-0.80$ (Table 1 ). The corresponding lower limit on the minor merger fraction ranges from $3.6 \%$ to $7.5 \%$. This is the first, albeit approximate, empirical estimate of the frequency of minor mergers over the last 7 Gyr.

For an assumed visibility timescale of $\sim 0.5 \mathrm{Gyr}$, it follows that over $T_{\mathrm{b}} \sim 3-7 \mathrm{Gyr}, \sim 68 \%$ of high-mass systems have undergone a merger of mass ratio $>1 / 10$, with $\sim 16 \%$, $45 \%$, and $7 \%$ of these corresponding respectively to major, minor, and ambiguous "major or minor" mergers. The corresponding merger rate $R$ is a few $\times 10^{-4}$ galaxies $\mathrm{Gyr}^{-1}$ $\mathrm{Mpc}^{-3}$.

2. At intermediate masses $\left(M \geqslant 1 \times 10^{9} M_{\odot}\right)$, we are only complete in mass for the blue cloud. Among $~ 2840$ bluecloud galaxies of mass $M \geqslant 1.0 \times 10^{9} M_{\odot}$, the fraction of visually classified systems that show evidence of a recent merger of mass ratio $>1 / 10$, ranges from $7 \% \pm 2 \%$ to $15 \%$ $\pm 5 \%$ over $z \sim 0.24-0.80$ (Table 2 ).

For an assumed visibility time of $\sim 0.5 \mathrm{Gyr}$, we estimate that on average, over $T_{\mathrm{b}} \sim 3-7 \mathrm{Gyr}, 84 \%$ of intermediatemass blue-cloud galaxies have undergone a merger of mass ratio $>1 / 10$, with $\sim 5 \%, 22 \%$, and $57 \%$ corresponding respectively to major, minor, and ambiguous "major or minor" mergers. The corresponding merger rate $R$ ranges from $8 \times 10^{-4}$ to $1 \times 10^{-3}$ galaxies $\mathrm{Gyr}^{-1} \mathrm{Mpc}^{-3}$. 
3. We compare our visual mergers to those identified using the widely used CAS merger criterion $(A>0.35$ and $A>S)$, based on CAS asymmetry $A$ and clumpiness $S$ parameters (Section 4.2). The merger fraction based on the CAS merger criterion agrees within a factor of 2 with the visually based merger fraction for high-mass $\left(M \geqslant 2.5 \times 10^{10} M_{\odot}\right)$ galaxies. However, for intermediate-mass $\left(M \geqslant 1 \times 10^{9}\right.$ $\left.M_{\odot}\right)$ galaxies, CAS can overestimate the merger fraction at $z>0.5$ by a factor $\sim 3$. In effect, over $z \sim 0.24-0.80$, $\sim 50 \%-70 \%$ of the galaxies visually classified as mergers satisfy the CAS criterion, but the latter also picks up a dominant number of non-interacting dusty, star-forming galaxies (Figures 7 and 8). These non-interacting systems make up as much as $\sim 45 \%-80 \%$ of the systems picked up the CAS criterion. We thus conclude that the traditional CAS merger criterion is ill-suited for use on $H S T V$-band images at $z>0.5$ where the rest frame wavelength falls below $\lambda<4000 \AA$, particularly in the case of intermediatemass galaxies with significant SF, gas, and dust. Modified CAS criteria in the near-UV based on morphological kcorrections (Taylor-Mager et al. 2007) might alleviate this problem.

4. We compare our empirical merger fraction $f$ and merger rate $R$ for high-mass $\left(M \geqslant 2.5 \times 10^{10} M_{\odot}\right)$ galaxies to predictions from different $\Lambda \mathrm{CDM}$-based simulations of galaxy evolution, including the halo occupation distribution (HOD) models of Hopkins et al. (2008); SAMs of Somerville et al. (2008), Bower et al. (2006), and Khochfar \& Silk (2006); and smoothed particle hydrodynamics (SPH) cosmological simulations from Maller et al. (2006) with a corrected stellar mass function (see Section 4.5). To our knowledge, such extensive comparisons have not been attempted to date, and are long overdue. We find qualitative agreement between the observations and models, such that the (major+minor) merger fraction (Figure 11) and merger rate (Figure 12) from different models bracket the corresponding empirical estimates and show a factor of 5 dispersion. One can now anticipate that in the near future, improvements in both the observational estimates and model predictions will start to rule out certain merger scenarios and refine our understanding of the merger history of galaxies.

5. We explore the impact of galaxy mergers on the SF activity of galaxies since $z<0.8$. In the sample of $\sim 789$ high-mass $\left(M \geqslant 2.5 \times 10^{10} M_{\odot}\right)$ galaxies, as well as the sample of $\sim 3600$ intermediate-mass $\left(M \geqslant 1.0 \times 10^{9} M_{\odot}\right)$ galaxies, we find that the average SFR of visibly merging galaxies is only modestly enhanced compared to non-interacting galaxies over $z \sim 0.24-0.80$ (Figure 14). This result is found for SFRs based on UV, UV+IR, as well as UV+stacked-IR data. This modest enhancement is consistent with the empirical results of Robaina et al. (2009, in preparation), and the recent statistical study of di Matteo et al. (2007) based on numerical simulations of several hundreds of galaxy collisions.

6. Among both high-mass and intermediate-mass galaxies, our results of a modest merger fraction $f$ and a modest enhancement in the average SFR due to mergers, culminate in our finding that visibly merging systems only account for a small fraction $(<30 \%)$ of the cosmic SFR density over lookback times of $\sim 3-7$ Gyr $(z \sim 0.24-0.80$; Figures 16 and 17). Our result complements that of Wolf et al. (2005) over a smaller lookback time interval of $\sim 6.2-6.8 \mathrm{Gyr}$.
In effect, our result suggests that the behavior of the cosmic SFR density over the last 7 Gyr is predominantly shaped by non-interacting galaxies, rather than mergers and interacting galaxies. We suggest that our observed decline in the cosmic SFR density by a factor of $\sim 3$ since $z \sim 0.80$ is largely the result of a shutdown in the SF of relatively non-interacting galaxies. This shutdown may be driven by the depletion of the internal cold gas supply of galaxies, the reduction in the accretion rate of gas from cosmological filaments, and the transition of SF activity to lower mass systems.

S.J. thanks Phil Hopkins, Sadegh Khochfar, Andrew Benson, Andi Burkert, and Ari Maller for useful discussions. S.J. acknowledges support from the National Aeronautics and Space Administration (NASA) LTSA grant NAG5-13063, NSF grant AST-0607748, and HST grants G0-10395 from STScI, which is operated by AURA, Inc., for NASA, under NAS526555. E.F.B., K.J., and A.R.R. acknowledge support from the Deutsche Forschungsgemeinschaft through the Emmy Noether Programme. X.Z.Z. acknowledges support from NSFC through grant 10773030,10833006. D.H.M. acknowledges support from NASA LTSA grant NAG5-13102 issued through the office of Space Science. C.Y.P. is grateful for support provided through STScI and NRC-HIA Fellowship. C.W. acknowledges support from an STFC Advanced Fellowship. Support for GEMS was provided by NASA through $H S T$ grant GO-9500 from the Space Telescope Science Institute, which is operated by AURA, Inc., for NASA, under NAS5-26555. This research has made use of NASA's Astrophysics Data System Service.

\section{REFERENCES}

Alexander, D. M., et al. 2003, AJ, 126, 539

Allam, S. S., Tucker, D. L., Smith, J. A., Lee, B. C., Annis, J., Lin, H., Karachentsev, I. D., \& Laubscher, B. E. 2004, AJ, 127, 1883

Barazza, F. D., Jogee, S., \& Marinova, I. 2008, ApJ, 675, 1194

Barden, M., Jahnke, K., \& Haussler, B. 2008, ApJS, 175, 105

Barden, M., et al. 2005, ApJ, 635, 959

Barnes, J. E., \& Hernquist, L. 1996, ApJ, 471, 115

Barnes, J. E., \& Hernquist, L. E. 1991, ApJL, 370, L65

Barton Gillespie, E., Geller, M. J., \& Kenyon, S. J. 2003, ApJ, 582, 668

Bauer, A. E., Drory, N., Hill, G. J., \& Feulner, G. 2005, ApJ, 621, L89

Bell, E. F., \& de Jong, R. S. 2001, ApJ, 550, 212

Bell, E. F., Phleps, S., Somerville, R. S., Wolf, C., Borch, A., \& Meisenheimer, K. 2006, ApJ, 652, 270

Bell, E. F., Zheng, X. Z., Papovich, C., Borch, A., Wolf, C., \& Meisenheimer, K. 2007, ApJ, 663, 834

Bell, E. F., et al. 2004, ApJ, 600, L11

Bell, E. F., et al. 2005, ApJ, 625, 23

Benson, A. J., Kamionkowski, M., \& Hassani, S. H. 2005, MNRAS, 357, 847 Bershady, M. A., Jangren, A., \& Conselice, C. J. 2000, AJ, 119, 2645

Binney, J., \& Merrifield, M. 1998, Galactic Astronomy (Princeton, NJ: Princeton Univ. Press)

Borch, A., et al. 2006, A\&A, 453, 869

Bower, R. G., Benson, A. J., Malbon, R., Helly, J. C., Frenk, C. S., Baugh, C. M., Cole, S., \& Lacey, C. G. 2006, MNRAS, 370, 645

Boylan-Kolchin, M., Ma, C.-P., \& Quataert, E. 2008, MNRAS, 383, 93

Brand, K., et al. 2006, ApJ, 644, 143

Brand, K., et al. 2006, ApJ, 641, 140

Brinchmann, J., Charlot, S., White, S. D. M., Tremonti, C., Kauffmann, G., Heckman, T., \& Brinkmann, J. 2004, MNRAS, 351, 1151

Brinchmann, J., \& Ellis, R. S. 2000, ApJ, 536, L77

Cassata, P., et al. 2005, MNRAS, 357, 903

Caldwell, J. A. R., et al. 2008, ApJS, 174, 136

Chabrier, G. 2003, PASP, 115, 763

Cole, S., Lacey, C. G., Baugh, C. M., \& Frenk, C. S. 2000, MNRAS, 319, 168

Conselice, C. J. 2003, ApJS, 147, 1

Conselice, C. J. 2006, ApJ, 638, 686 
Conselice, C. J., Bershady, M. A., Dickinson, M., \& Papovich, C. 2003, AJ, 126,1183

Conselice, C. J., Bershady, M. A., \& Jangren, A. 2000, ApJ, 529, 886

Conselice, C. J., Blackburne, J. A., \& Papovich, C. 2005, ApJ, 620, 564

Conselice, C. J., Rajgor, S., \& Myers, R. 2008, MNRAS, 386, 909

Cowie, L. L., Songaila, A., Hu, E. M., \& Cohen, J. G. 1996, AJ, 112, 839

Cox, T. J., Jonsson, P., Primack, J. R., \& Somerville, R. S. 2006, MNRAS, 373, 1013

Croton, D. J., et al. 2006, MNRAS, 367, 864

de Ravel, L., et al. 2008, A\&A, in press (arXiv:0807.2578)

Devriendt, J. E. G., Guiderdoni, B., \& Sadat, R. 1999, A\&A, 350, 381

di Matteo, P., Combes, F., Melchior, A.-L., \& Semelin, B. 2007, A\&A, 468, 6

D’Onghia, E., \& Burkert, A. 2004, ApJ, 612, L13

D’Onghia, E., Mapelli, M., \& Moore, B. 2008, MNRAS, 389, 1275

Ellis, R. S., Colless, M., Broadhurst, T., Heyl, J., \& Glazebrook, K. 1996, MNRAS, 280, 235

Fakhouri, O., \& Ma, C.-P. 2008, MNRAS, 386, 577

Fioc, M., \& Rocca-Volmerange, B. 1997, A\&A, 326, 950

Flores, H., et al. 1999, ApJ, 517, 148

Fontana, A., et al. 2003, ApJ, 594, L9

Franceschini, A., et al. 2005, AJ, 129, 2074

Gordon, K. D., et al. 2005, PASP, 117, 503

Gottlöber, S., Klypin, A., \& Kravtsov, A. V. 2001, ApJ, 546, 223

Governato, F., et al. 2004, ApJ, 607, 688

Haarsma, D. B., Partridge, R. B., Windhorst, R. A., \& Richards, E. A. 2000, ApJ, 544,641

Hammer, F., Flores, H., Elbaz, D., Zheng, X. Z., Liang, Y. C., \& Cesarsky, C. 2005, A\&A, 430, 115

Hernquist, L. 1989, Nature, 340, 687

Hernquist, L., \& Mihos, J. C. 1995, ApJ, 448, 41

Hopkins, A. M. 2004, ApJ, 615, 209

Hopkins, P. F., Somerville, R. S., Hernquist, L., Cox, T. J., Robertson, B., \& Li, Y. 2006, ApJ, 652, 864

Hopkins, P. F., et al. 2008, ApJS, 175, 356

Jogee, S. 1999, Ph.D. thesis, Yale Univ.

Jogee, S. 2006, in Lecture Notes in Physics 693, AGN Physics on All Scales, Vol. 93, ed. D. Alloin, R. Johnson, \& P. Lira, (chapter 6; Berlin: Springer), 143 (arXiv:astro-ph/0408383)

Jogee, S., Kenney, J. D. P., \& Smith, B. J. 1998, ApJ, 494, L185

Jogee, S., Kenney, J. D. P., \& Smith, B. J. 1999, ApJ, 526, 665

Jogee, S., et al. 2008, ASP Conf. Ser. 396, Formation and Evolution of Galaxy Disks, ed. J. G. Funes \& E. M. Corsini (San Francisco, CA: ASP), 337

Joseph, R. D., \& Wright, G. S. 1985, MNRAS, 214, 87

Kartaltepe, J. S., et al. 2007, ApJS, 172, 320

Kauffmann, G., White, S. D. M., \& Guiderdoni, B. 1993, MNRAS, 264, 201

Kautsch, S. J., Grebel, E. K., Barazza, F. D., \& Gallagher, J. S., III. 2006, A\&A, 451,1171

Kennicutt, R. C., Jr., Roettiger, K. A., Keel, W. C., van der Hulst, J. M., \& Hummel, E. 1987, AJ, 93, 1011

Khochfar, S., \& Burkert, A. 2005, MNRAS, 359, 1379

Khochfar, S., \& Silk, J. 2006, MNRAS, 370, 902

Kroupa, P. 2001, MNRAS, 322, 231

Kroupa, P., Tout, C. A., \& Gilmore, G. 1993, MNRAS, 262, 545

Lacey, C., \& Cole, S. 1993, MNRAS, 262, 627

Larson, R. B., \& Tinsley, B. M. 1978, ApJ, 219, 46

Le Floc'h, E., et al. 2005, ApJ, 632, 169

Le Fèvre, O., et al. 2000, MNRAS, 311, 565
Lehmer, B. D., et al. 2005, ApJS, 161, 21

Lilly, S. J., Le Fevre, O., Hammer, F., \& Crampton, D. 1996, ApJ, 460, L1

Lin, L., et al. 2004, ApJ, 617, L9

Lotz, J. M., Primack, J., \& Madau, P. 2004, AJ, 128, 163

Lotz, J. M., et al. 2008, ApJ, 672, 177

Maller, A. H., Katz, N., Kereš, D., Davé, R., \& Weinberg, D. H. 2006, ApJ, 647, 763

Melbourne, J., Koo, D. C., \& Le Floc'h, E. 2005, ApJ, 632, L65

Mihos, J. C., \& Hernquist, L. 1994, ApJ, 437, 611

Mihos, J. C., \& Hernquist, L. 1996, ApJ, 464, 641

Mihos, J. C., Walker, I. R., Hernquist, L., Mendes de Oliveira, C., \& Bolte, M. 1995, ApJ, 447, L87

Miller, S. H., et al. 2008, in Frank N. Bash Symp. 393, New Horizons in Astronomy, ed. A. Frebel, J. Maund, J. Shen, \& M. Siegel (San Fransisco, CA: ASP), 235

Mobasher, B., Jogee, S., Dahlen, T., de Mello, D., Lucas, R. A., Conselice, C. J., Grogin, N. A., \& Livio, M. 2004, ApJ, 600, L143

Murali, C., Katz, N., Hernquist, L., Weinberg, D. H., \& Davé, R. 2002, ApJ, 571,1

Naab, T., \& Burkert, A. 2001, ApJ, 555, L91

Navarro, J. F., \& Benz, W. 1991, ApJ, 380, 320

Navarro, J. F., \& Steinmetz, M. 2000, ApJ, 538, 477

Negroponte, J., \& White, S. D. M. 1983, MNRAS, 205, 1009

Neistein, E., \& Dekel, A. 2008, MNRAS, 388, 1792

Noeske, K. G., et al. 2007a, ApJ, 660, 43

Noeske, K. G., et al. 2007b, ApJ, 660, L47

Papovich, C., \& Bell, E. F. 2002, ApJ, 579, L1

Papovich, C., et al. 2004, ApJS, 154, 70

Patton, D. R., Carlberg, R. G., Marzke, R. O., Pritchet, C. J., da Costa, L. N., \& Pellegrini, P. S. 2000, ApJ, 536, 153

Pérez-González, P. G., et al. 2005, ApJ, 630, 8

Quinn, P. J., Hernquist, L., \& Fullagar, D. P. 1993, ApJ, 403, 74

Rieke, G. H., et al. 2004, ApJS, 154, 25

Rix, H.-W., et al. 2004, ApJS, 152, 163

Robertson, B., Yoshida, N., Springel, V., \& Hernquist, L. 2004, ApJ, 606, 32

Silva, L., Maiolino, R., \& Granato, G. L. 2004, MNRAS, 355, 973

Sobral, D., et al. 2009, MNRAS, submitted (arXiv:0901.4114v1)

Smith, B. J. 1994, AJ, 107, 1695

Smith, B. J. 1997, AJ, 114, 2177

Somerville, R. S., Hopkins, P. F., Cox, T. J., Robertson, B. E., \& Hernquist, L. 2008, MNRAS, 391, 481

Somerville, R. S., \& Kolatt, T. S. 1999, MNRAS, 305, 1

Somerville, R. S., \& Primack, J. R. 1999, MNRAS, 310, 1087

Springel, V., et al. 2005a, Nature, 435, 629

Springel, V., Di Matteo, T., \& Hernquist, L. 2005b, MNRAS, 361, 776

Struck, C. 1997, ApJS, 113, 269

Taylor-Mager, V. A., Conselice, C. J., Windhorst, R. A., \& Jansen, R. A 2007, ApJ, 659, 162

van den Bosch, F. C., et al. 2007, MNRAS, 376, 841

Weinzirl, T., Jogee, S., Khochfar, S., Burkert, A., \& Kormendy, J. 2009, ApJ, 696, 411

Wolf, C., et al. 2004, A\&A, 421, 913

Wolf, C., et al. 2005, ApJ, 630, 771

Zheng, X. Z., Bell, E. F., Papovich, C., Wolf, C., Meisenheimer, K., Rix, H.-W., Rieke, G. H., \& Somerville, R. 2007, ApJ, 661, L41

Zheng, X. Z., et al. 2006, ApJ, 640, 784 